Supporting Information for

\title{
Parent Cyclopentadienyl Ruthenium(II) Chloride Synthon: Derivatization to CpRu Amido, Imido, and Oxo Complexes
}

\author{
Shin Takemoto, ${ }^{*}$ Hana Ishii, Masahiro Yamaguchi, Akira Teramoto, Masayuki Tsujita, \\ Daiki Ozeki, and Hiroyuki Matsuzaka* \\ Department of Chemistry, Graduate School of Science, Osaka Prefecture University
}

\section{Contents}

Figure S1. ${ }^{1} \mathrm{H}$ NMR spectrum of [1][2] (400 MHz, $\left.\mathrm{CD}_{3} \mathrm{NO}_{2}\right)$.

Figure S2. ${ }^{13} \mathrm{C}\left\{{ }^{1} \mathrm{H}\right\}$ NMR spectrum of $[1][2]\left(100 \mathrm{MHz}, \mathrm{CD}_{3} \mathrm{NO}_{2}\right) . \quad$ S2

Figure S3. $\quad{ }^{1} \mathrm{H}$ NMR spectrum of $\mathbf{3}\left(400 \mathrm{MHz}, \mathrm{CDCl}_{3}\right) . \quad \mathrm{S} 3$

Figure S4. ${ }^{13} \mathrm{C}\left\{{ }^{1} \mathrm{H}\right\}$ NMR spectrum of $\mathbf{3}\left(100 \mathrm{MHz}, \mathrm{CDCl}_{3}\right) . \quad \mathrm{S} 3$

Figure S5. $\quad{ }^{1} \mathrm{H}$ NMR spectrum of $4\left(400 \mathrm{MHz}, \mathrm{CD}_{2} \mathrm{Cl}_{2}\right) . \quad \mathrm{S} 4$

Figure S6. ${ }^{13} \mathrm{C}\left\{{ }^{1} \mathrm{H}\right\}$ NMR spectrum of $4\left(100 \mathrm{MHz}, \mathrm{CD}_{2} \mathrm{Cl}_{2}\right) . \quad \mathrm{S} 4$

Figure S7. ${ }^{1} \mathrm{H}$ NMR spectrum of $5\left(400 \mathrm{MHz}, \mathrm{CDCl}_{3}\right)$. S5

Figure S8. $\quad{ }^{1} \mathrm{H}$ NMR spectrum of $6\left(400 \mathrm{MHz}, \mathrm{CDCl}_{3}\right)$. S5

Figure S9. ${ }^{1} \mathrm{H}$ NMR spectrum of $7\left(400 \mathrm{MHz}, \mathrm{C}_{6} \mathrm{D}_{6}\right) . \quad$ S6

Figure S10. ${ }^{13} \mathrm{C}\left\{{ }^{1} \mathrm{H}\right\}$ NMR spectrum of $7\left(100 \mathrm{MHz}, \mathrm{C}_{6} \mathrm{D}_{6}\right) . \quad$ S6

Figure S11. $\quad{ }^{1} \mathrm{H}$ NMR spectrum of $8\left(400 \mathrm{MHz}, \mathrm{C}_{6} \mathrm{D}_{6}\right) . \quad$ S7

Figure S12. ${ }^{13} \mathrm{C}\left\{{ }^{1} \mathrm{H}\right\}$ NMR spectrum of $8\left(100 \mathrm{MHz}, \mathrm{C}_{6} \mathrm{D}_{6}\right) . \quad$ S7

Figure S13. ${ }^{1} \mathrm{H}$ NMR spectrum of $[9]\left[\mathrm{BF}_{4}\right]\left(400 \mathrm{MHz}, \mathrm{DMSO}-\mathrm{d}_{6}\right) . \quad$ S8

Figure S14. ${ }^{13} \mathrm{C}\left\{{ }^{1} \mathrm{H}\right\}$ NMR spectrum of $[9]\left[\mathrm{BF}_{4}\right]\left(100 \mathrm{MHz}, \mathrm{DMSO}-\mathrm{d}_{6}\right) . \quad \mathrm{S} 8$

Figure S15. ${ }^{1} \mathrm{H}$ NMR spectrum of $[9]\left[\mathrm{B}\left(\mathrm{C}_{6} \mathrm{~F}_{5}\right)_{4}\right]\left(400 \mathrm{MHz}, \mathrm{DMSO}-\mathrm{d}_{6}\right) . \quad$ S9

Figure S16. ${ }^{13} \mathrm{C}\left\{{ }^{1} \mathrm{H}\right\}$ NMR spectrum of $[9]\left[\mathrm{B}\left(\mathrm{C}_{6} \mathrm{~F}_{5}\right)_{4}\right]\left(100 \mathrm{MHz}\right.$, DMSO-d $\left.\mathrm{d}_{6}\right) . \quad$ S9

Figure S17. ${ }^{1} \mathrm{H}$ NMR spectrum of $[\mathbf{1 0}]\left[\mathrm{BF}_{4}\right]\left(400 \mathrm{MHz}, \mathrm{DMSO}-\mathrm{d}_{6}\right) . \quad \mathrm{S} 10$

Figure S18. ${ }^{13} \mathrm{C}\left\{{ }^{1} \mathrm{H}\right\}$ NMR spectrum of $[\mathbf{1 0}]\left[\mathrm{BF}_{4}\right](100 \mathrm{MHz}$, DMSO-d 6$) . \quad \mathrm{S} 10$

Figure S19. ${ }^{1} \mathrm{H}$ NMR spectrum of $\mathbf{1 1}\left(400 \mathrm{MHz}, \mathrm{CD}_{2} \mathrm{Cl}_{2}\right) . \quad \mathrm{S} 11$

Figure S20. ${ }^{13} \mathrm{C}\left\{{ }^{1} \mathrm{H}\right\}$ NMR spectrum of $\mathbf{1 1}\left(100 \mathrm{MHz}, \mathrm{CD}_{2} \mathrm{Cl}_{2}\right) . \quad \mathrm{S} 11$

Figure S21. ${ }^{1} \mathrm{H}$ NMR spectrum of $\mathbf{1 2}\left(400 \mathrm{MHz}, \mathrm{C}_{6} \mathrm{D}_{6}\right) . \quad \mathrm{S} 12$

Figure S22. ${ }^{13} \mathrm{C}\left\{{ }^{1} \mathrm{H}\right\}$ NMR spectrum of $\mathbf{1 2}\left(100 \mathrm{MHz}, \mathrm{C}_{6} \mathrm{D}_{6}\right) . \quad \mathrm{S} 12$

Figure S23. $\quad{ }^{1} \mathrm{H}$ NMR spectrum of $\mathbf{1 4}\left(400 \mathrm{MHz}, \mathrm{CDCl}_{3}\right) . \quad \mathrm{S} 13$

Figure S24. ${ }^{13} \mathrm{C}\left\{{ }^{1} \mathrm{H}\right\}$ NMR spectrum of $\mathbf{1 4}\left(100 \mathrm{MHz}, \mathrm{CDCl}_{3}\right) . \quad \mathrm{S} 13$

$\begin{array}{lll}\text { Figure S25. } & { }^{1} \mathrm{H} \text { NMR spectrum of } \mathbf{1 5}\left(400 \mathrm{MHz}, \mathrm{CDCl}_{3}\right) . & \mathrm{S} 14\end{array}$

$\begin{array}{lll}\text { Figure S26. } & { }^{13} \mathrm{C}\left\{{ }^{1} \mathrm{H}\right\} \text { NMR spectrum of } \mathbf{1 5}\left(100 \mathrm{MHz}, \mathrm{CDCl}_{3}\right) . & \mathrm{S} 14\end{array}$

Figure S27. $\mathrm{GC}$ for the gas phase analysis of the reaction of $\mathbf{1 4}$ with $\mathrm{CO} \quad \mathrm{S} 15$

Table S1. Crystallographic data for $[1][2] \cdot \mathrm{CH}_{2} \mathrm{Cl}_{2}, 3,[9]\left[\mathrm{B}\left(\mathrm{C}_{6} \mathrm{~F}_{5}\right)_{4}\right] \cdot \mathrm{C}_{6} \mathrm{H}_{14},\left[9^{*}\right]\left[\mathrm{B}\left(\mathrm{C}_{6} \mathrm{~F}_{5}\right)_{4}\right] . \quad \mathrm{S} 16$

Table S2. Crystallographic data for $[\mathbf{1 0}][\mathrm{BF} 4] \cdot \mathrm{THF}, \mathbf{1 1}, \mathbf{1 4} \cdot \mathrm{CH}_{2} \mathrm{Cl}_{2}$, and $\mathbf{1 5} \cdot \mathrm{CH}_{2} \mathrm{Cl}_{2}$. 


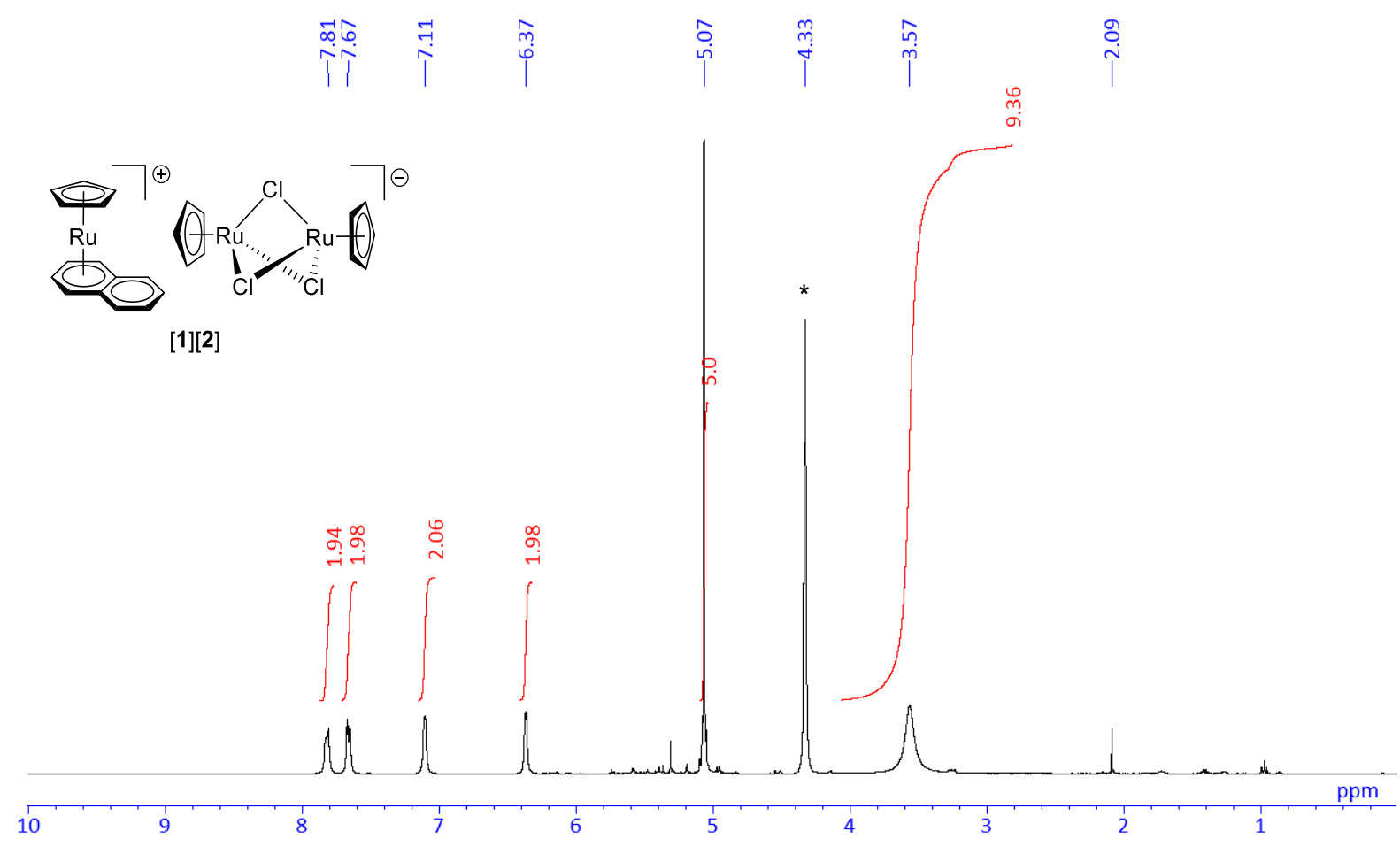

Figure S1. ${ }^{1} \mathrm{H}$ NMR spectrum of [1][2] $\left(400 \mathrm{MHz}, \mathrm{CD}_{3} \mathrm{NO}_{2}\right) . \quad$ *solvent peak

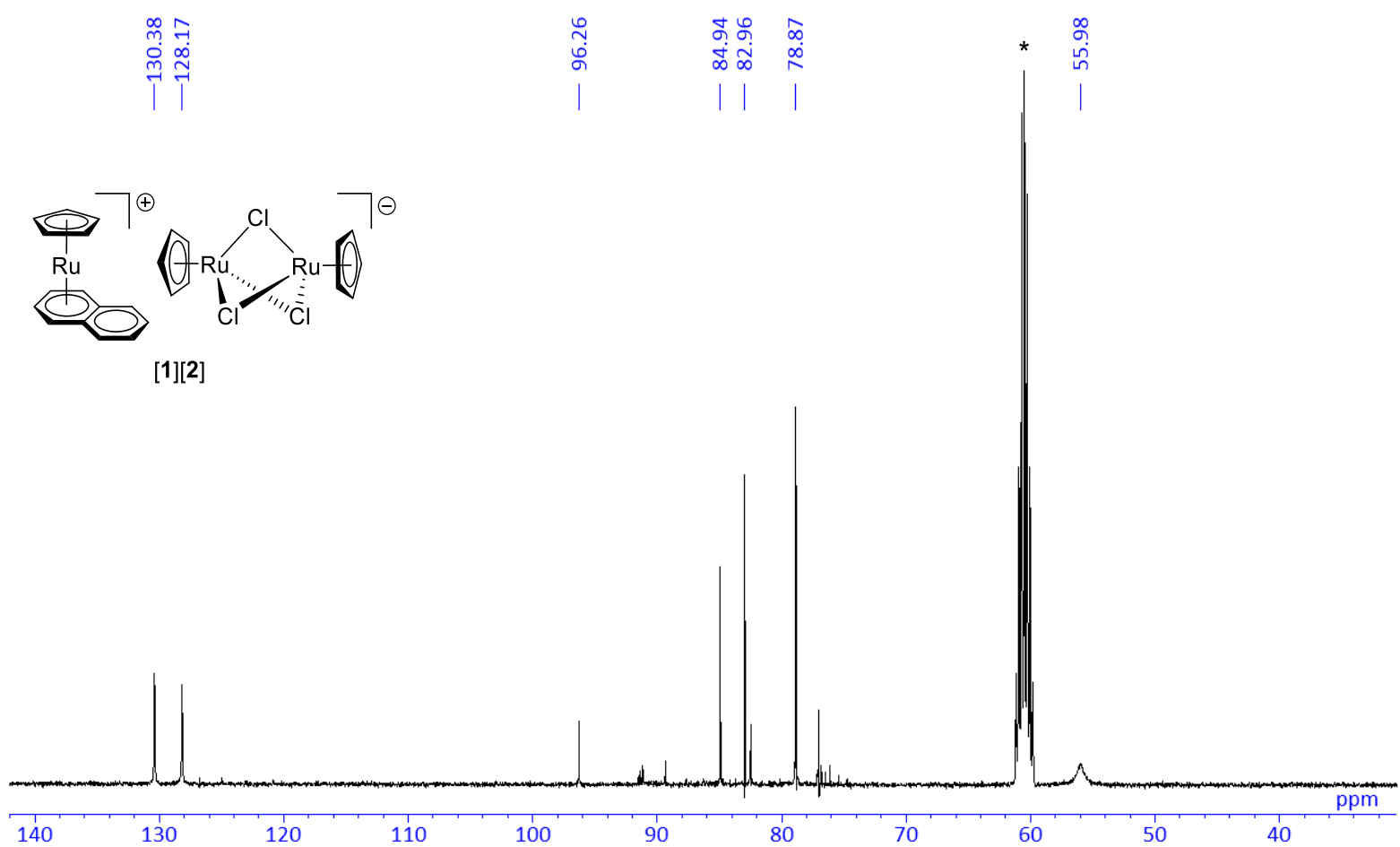

Figure S2. ${ }^{13} \mathrm{C}\left\{{ }^{1} \mathrm{H}\right\}$ NMR spectrum of $[1][2]\left(100 \mathrm{MHz}, \mathrm{CD}_{3} \mathrm{NO}_{2}\right) . \quad$ *solvent peak 


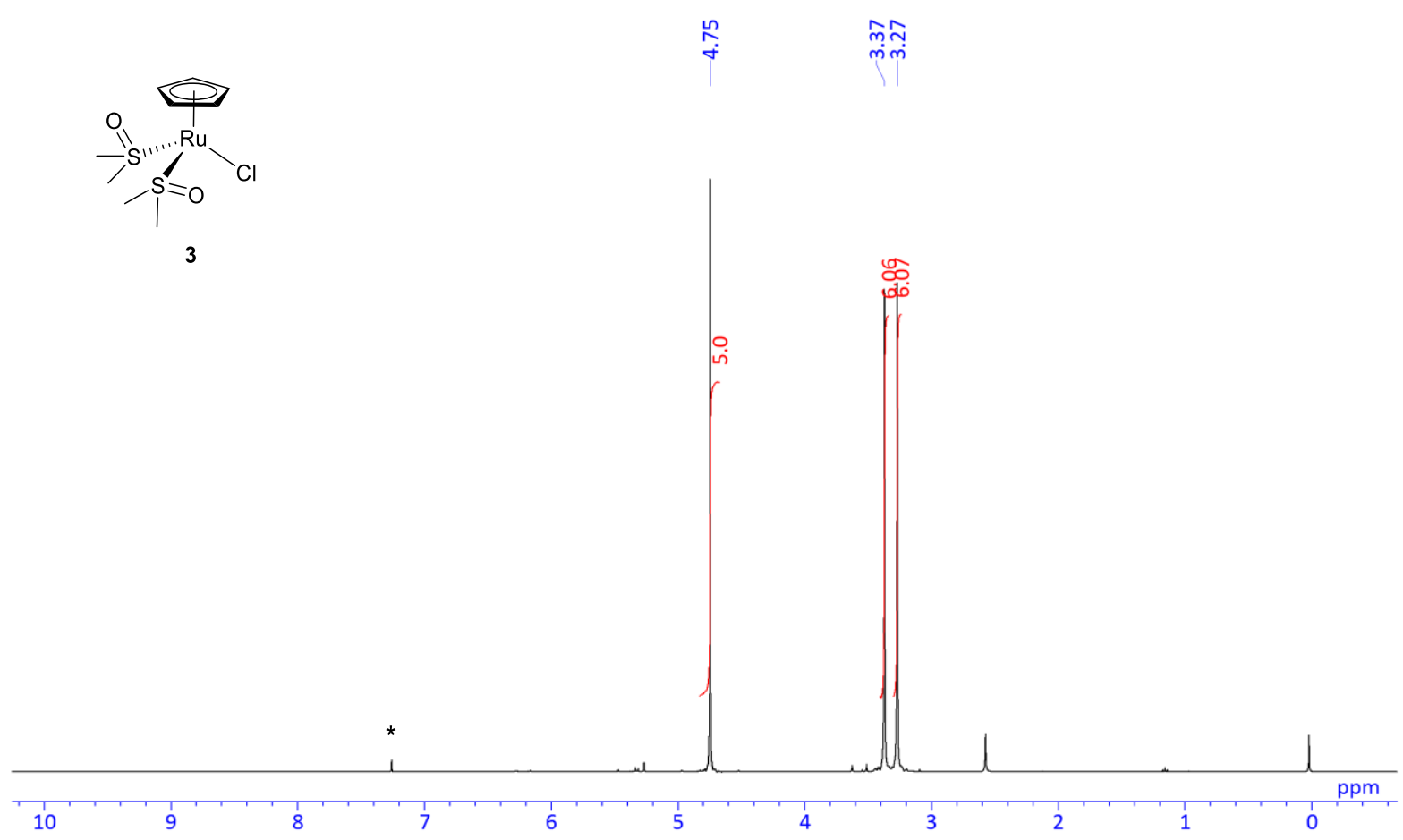

Figure S3. $\quad{ }^{1} \mathrm{H}$ NMR spectrum of $3\left(400 \mathrm{MHz}, \mathrm{CDCl}_{3}\right) . \quad$ *solvent peak

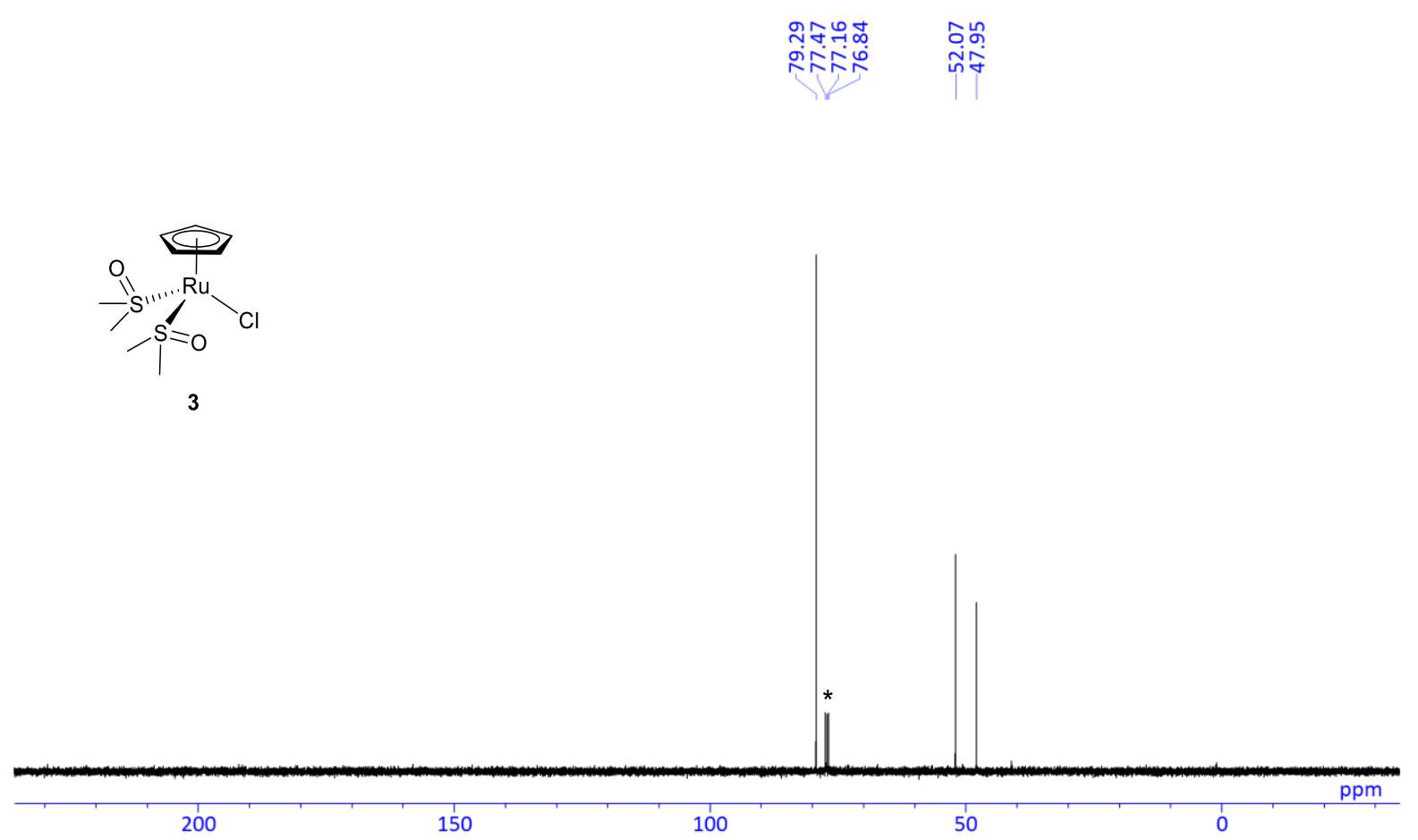

Figure S4. ${ }^{13} \mathrm{C}\left\{{ }^{1} \mathrm{H}\right\}$ NMR spectrum of $3\left(100 \mathrm{MHz}, \mathrm{CDCl}_{3}\right)$. *solvent peak 

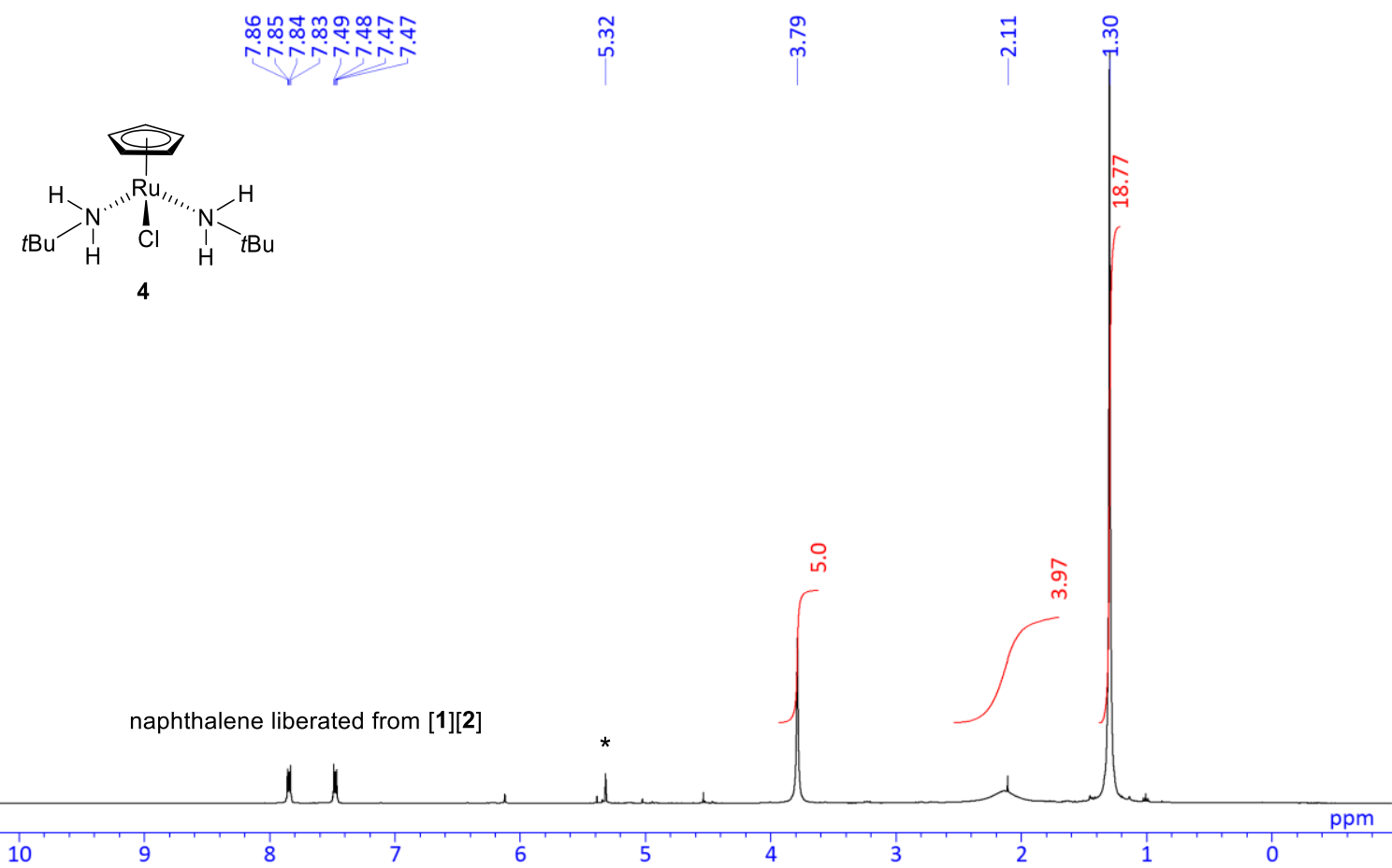

Figure S5. $\quad{ }^{1} \mathrm{H}$ NMR spectrum of $4\left(400 \mathrm{MHz}, \mathrm{CD}_{2} \mathrm{Cl}_{2}\right)$. $\quad{ }^{*}$ solvent peak

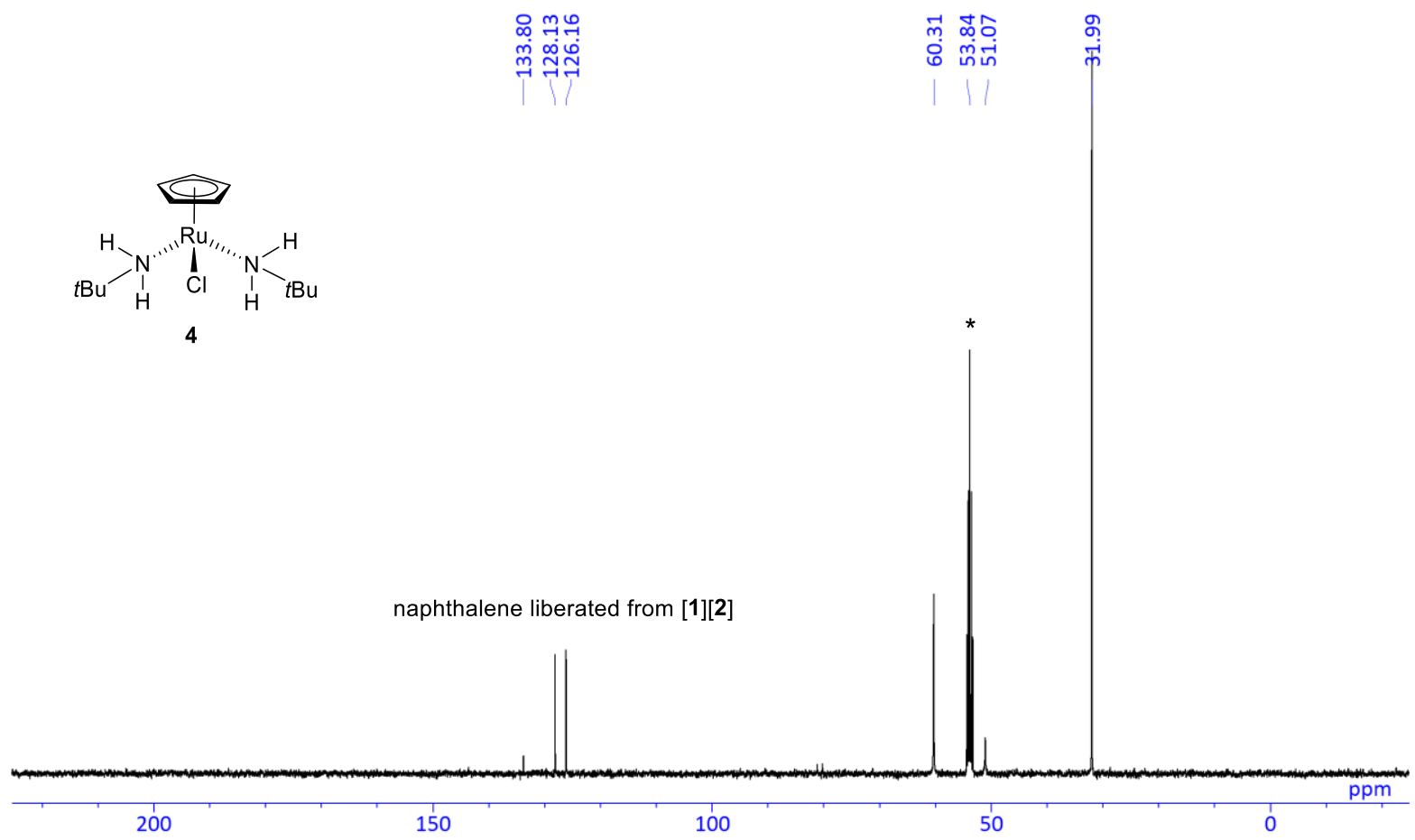

Figure S6. ${ }^{13} \mathrm{C}\left\{{ }^{1} \mathrm{H}\right\}$ NMR spectrum of $4\left(100 \mathrm{MHz}, \mathrm{CD}_{2} \mathrm{Cl}_{2}\right)$. * solvent peak 


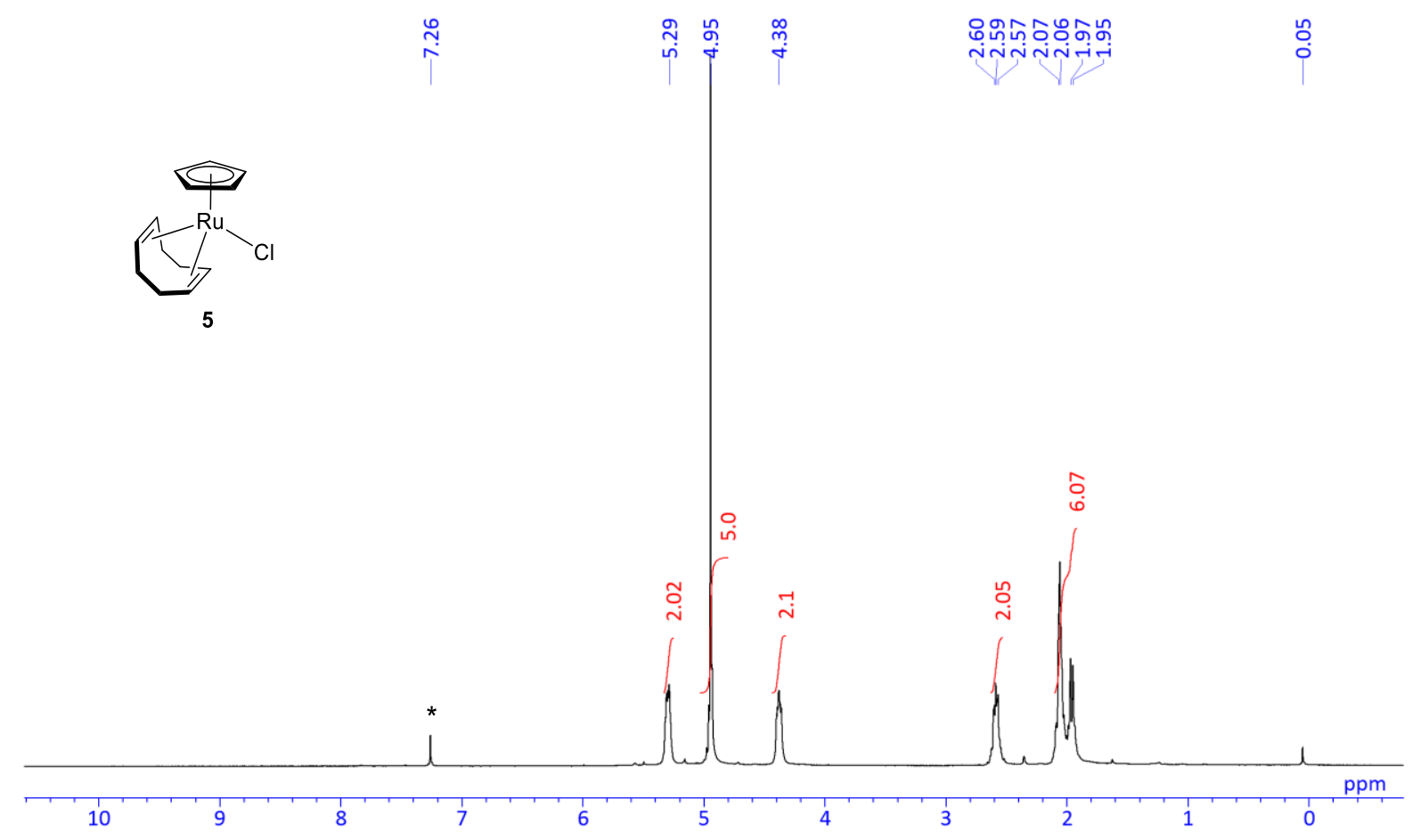

Figure S7. $\quad{ }^{1} \mathrm{H}$ NMR spectrum of $5\left(400 \mathrm{MHz}, \mathrm{CDCl}_{3}\right) . \quad$ *solvent peak

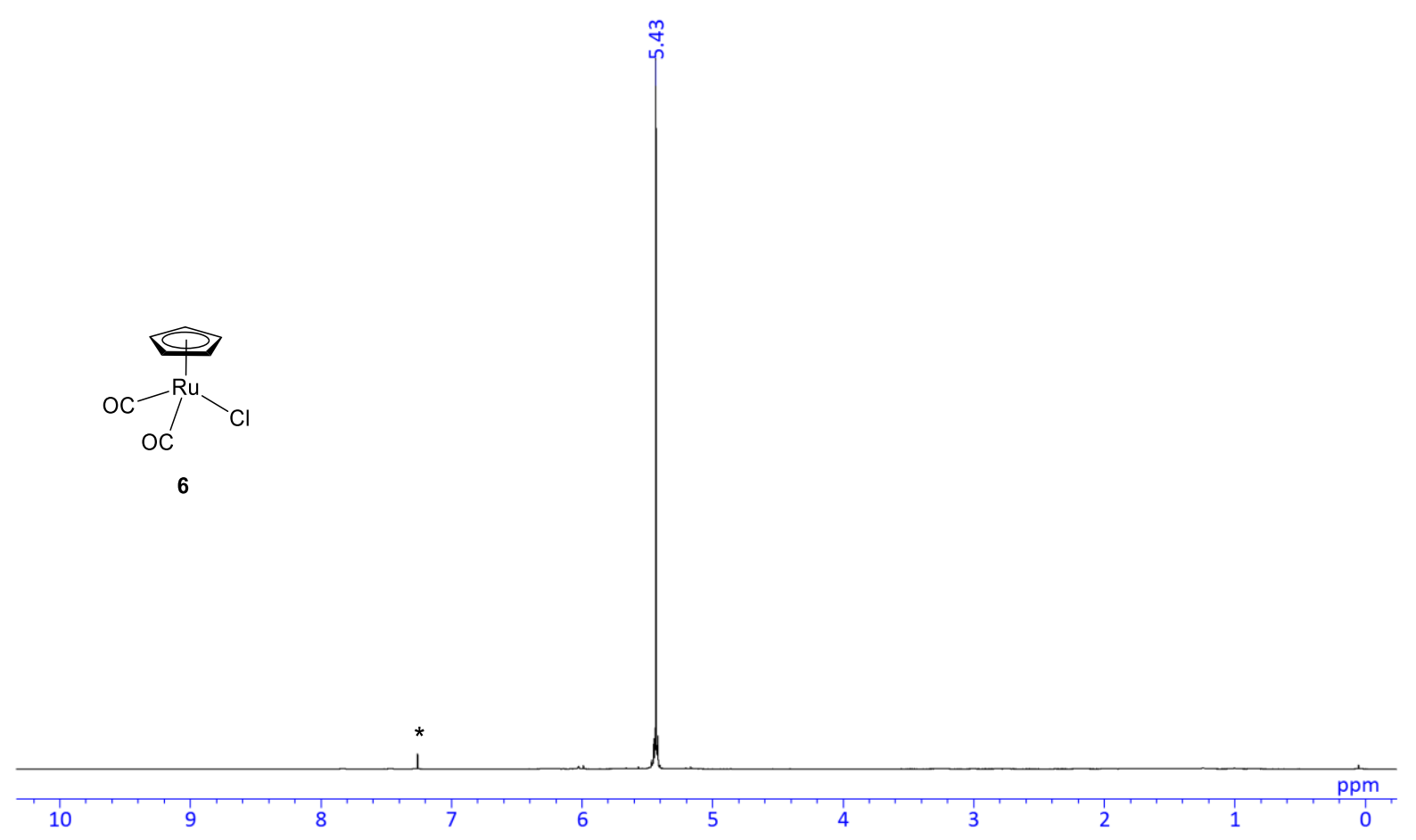

Figure S8. $\quad{ }^{1} \mathrm{H}$ NMR spectrum of $6\left(400 \mathrm{MHz}, \mathrm{CDCl}_{3}\right) . \quad$ *solvent peak 


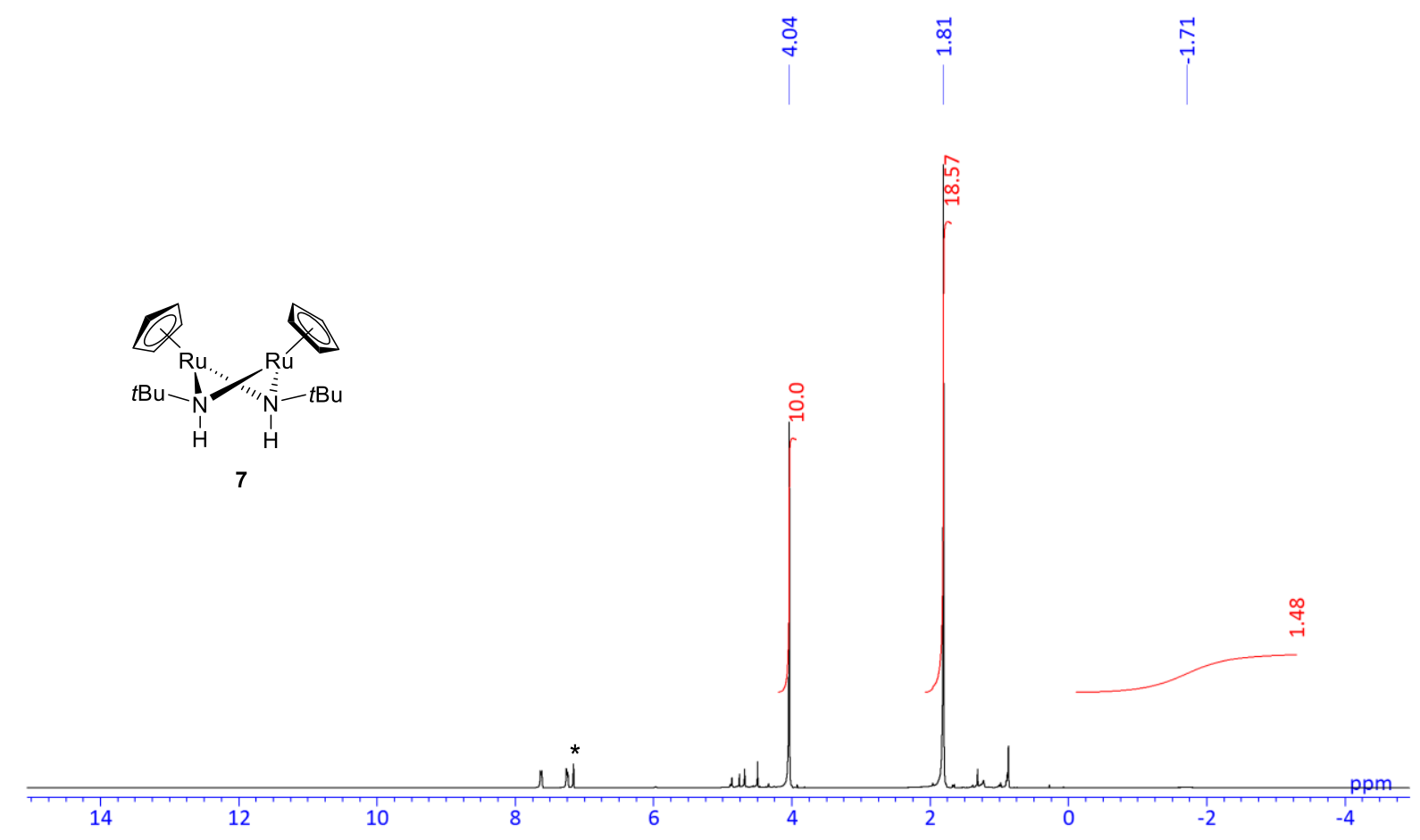

Figure S9. $\quad{ }^{1} \mathrm{H}$ NMR spectrum of $7\left(400 \mathrm{MHz}, \mathrm{C}_{6} \mathrm{D}_{6}\right)$. $\quad$ *solvent peak

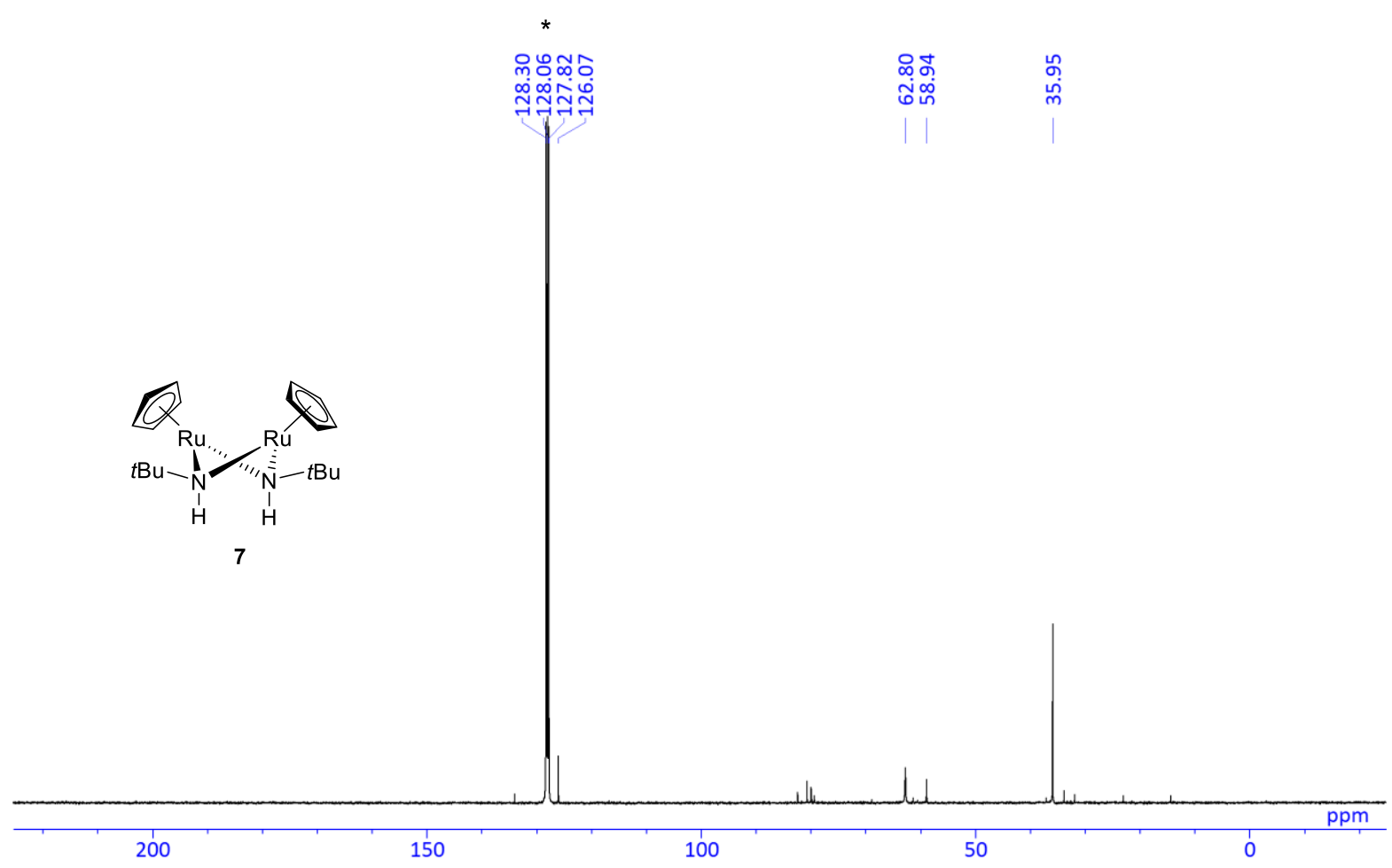

Figure S10. ${ }^{13} \mathrm{C}\left\{{ }^{1} \mathrm{H}\right\}$ NMR spectrum of $7\left(100 \mathrm{MHz}, \mathrm{C}_{6} \mathrm{D}_{6}\right) . \quad{ }^{*}$ solvent peak 


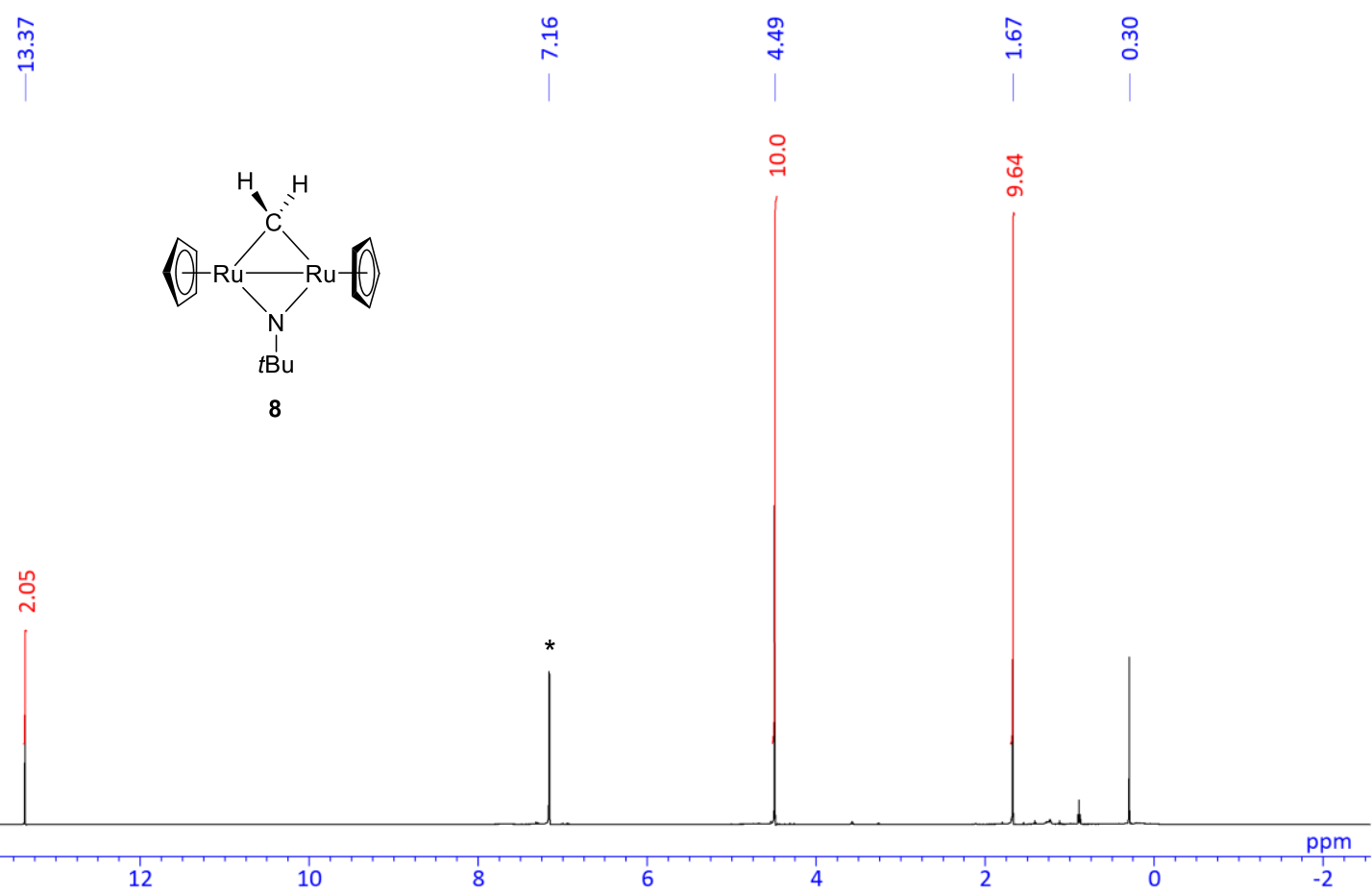

Figure S11. ${ }^{1} \mathrm{H}$ NMR spectrum of $8\left(400 \mathrm{MHz}, \mathrm{C}_{6} \mathrm{D}_{6}\right)$. * * solvent peak

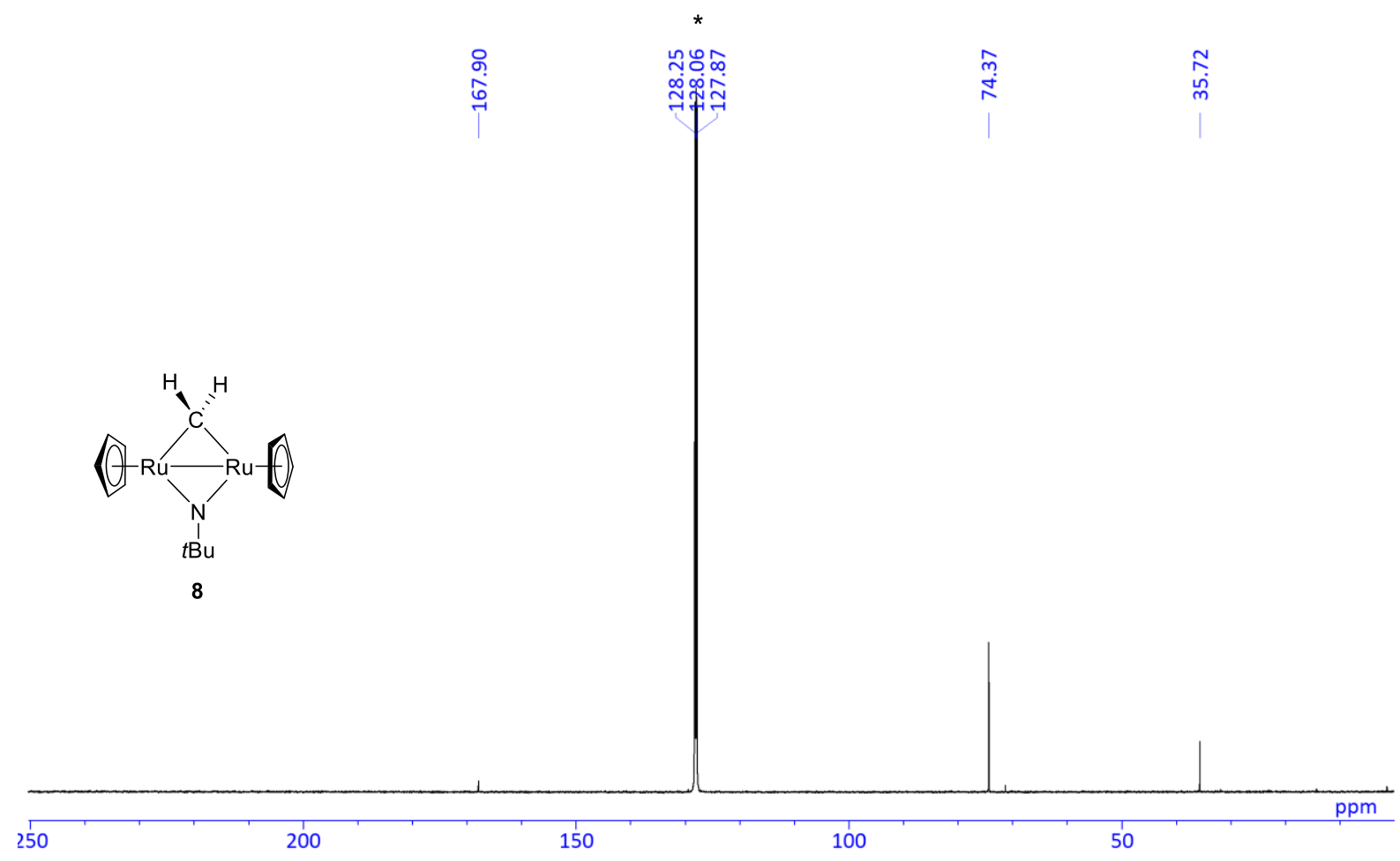

Figure S12. ${ }^{13} \mathrm{C}\left\{{ }^{1} \mathrm{H}\right\}$ NMR spectrum of $8\left(100 \mathrm{MHz}, \mathrm{C}_{6} \mathrm{D}_{6}\right)$. * solvent peak 


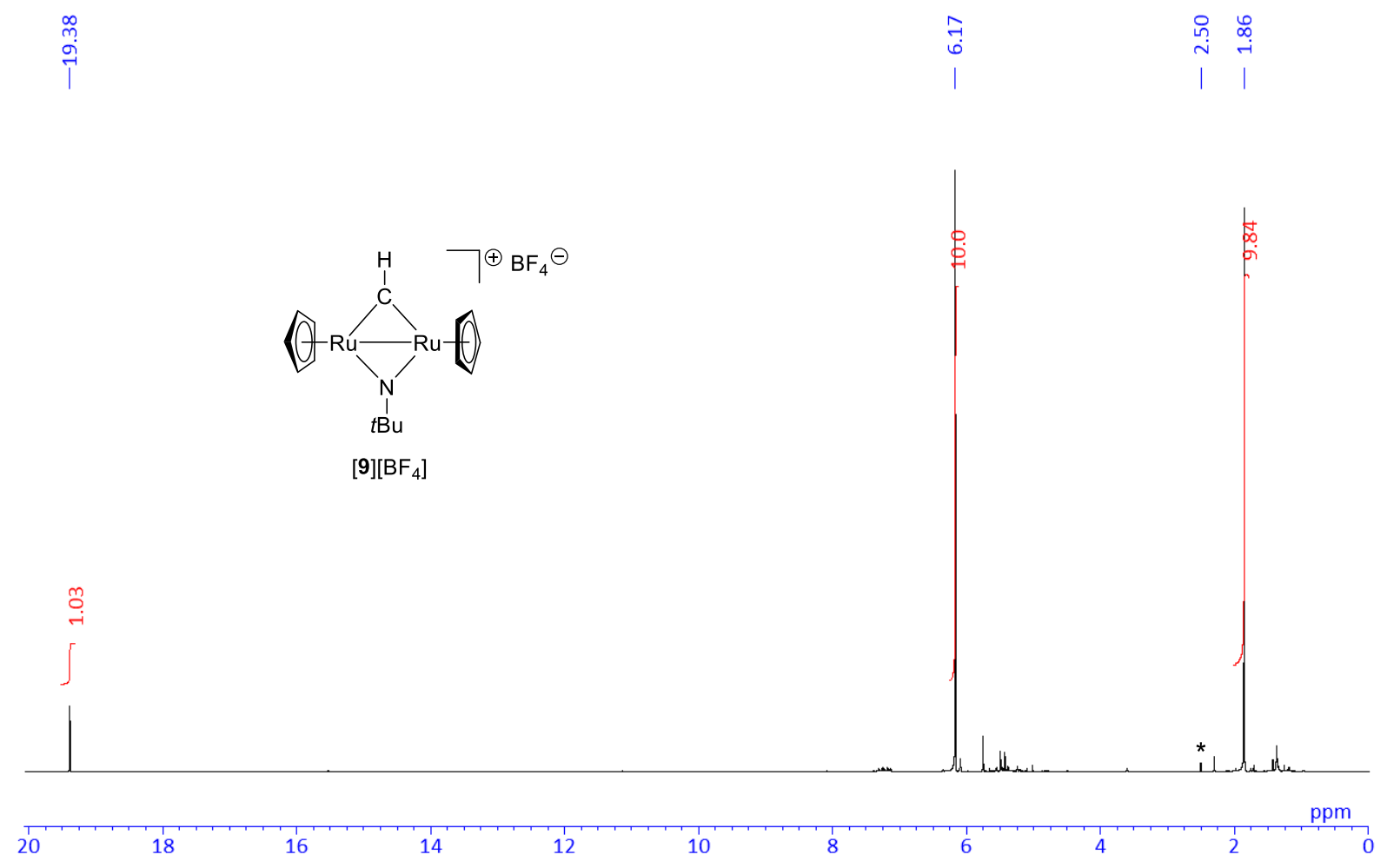

Figure S13. ${ }^{1} \mathrm{H}$ NMR spectrum of $[9]\left[\mathrm{BF}_{4}\right]\left(400 \mathrm{MHz}, \mathrm{DMSO}-\mathrm{d}_{6}\right) . \quad{ }^{*}$ solvent peak(s)

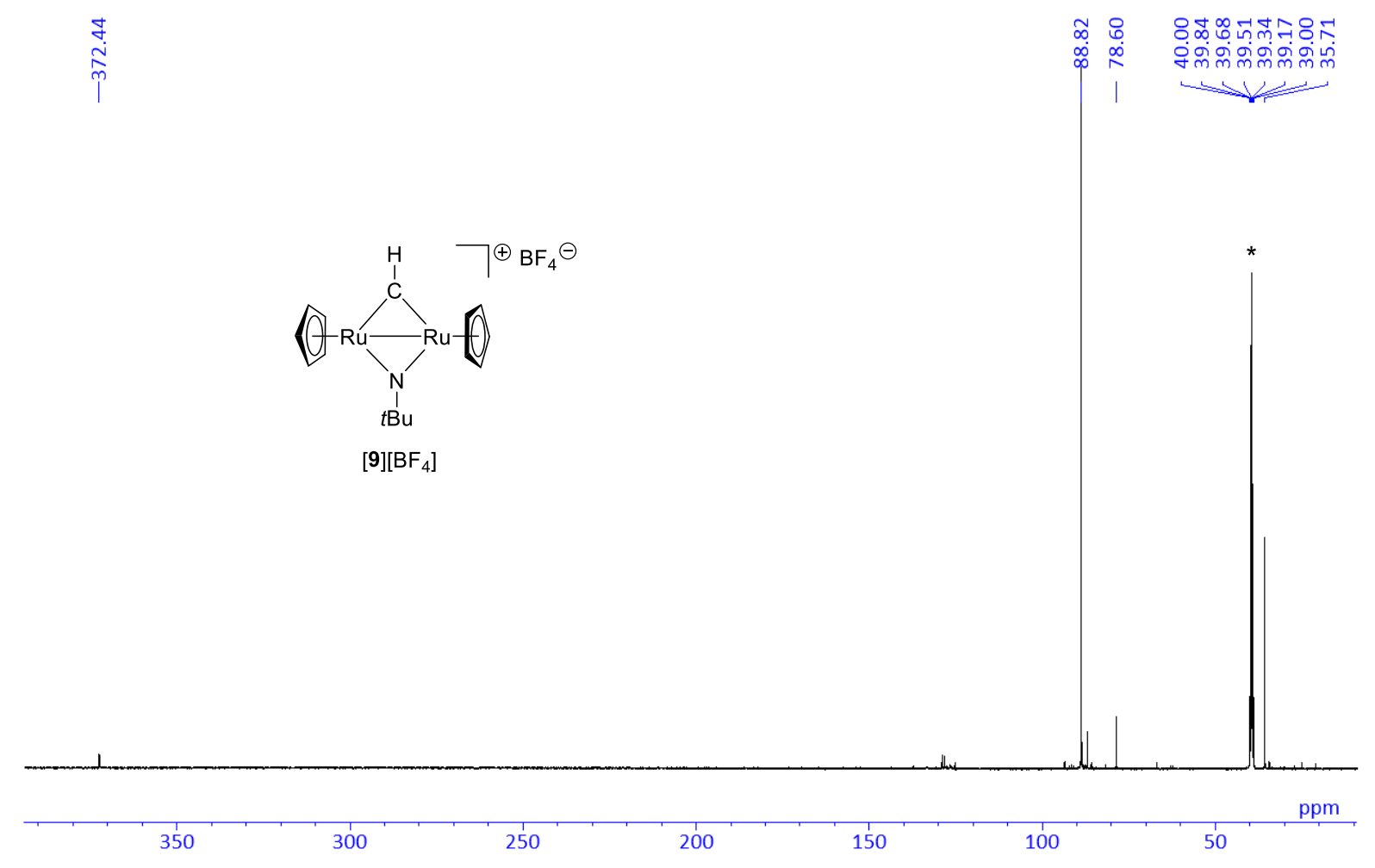

Figure S14. ${ }^{13} \mathrm{C}\left\{{ }^{1} \mathrm{H}\right\}$ NMR spectrum of $[9]\left[\mathrm{BF}_{4}\right]\left(100 \mathrm{MHz}, \mathrm{DMSO}-\mathrm{d}_{6}\right) . \quad{ }^{*}$ solvent peak 


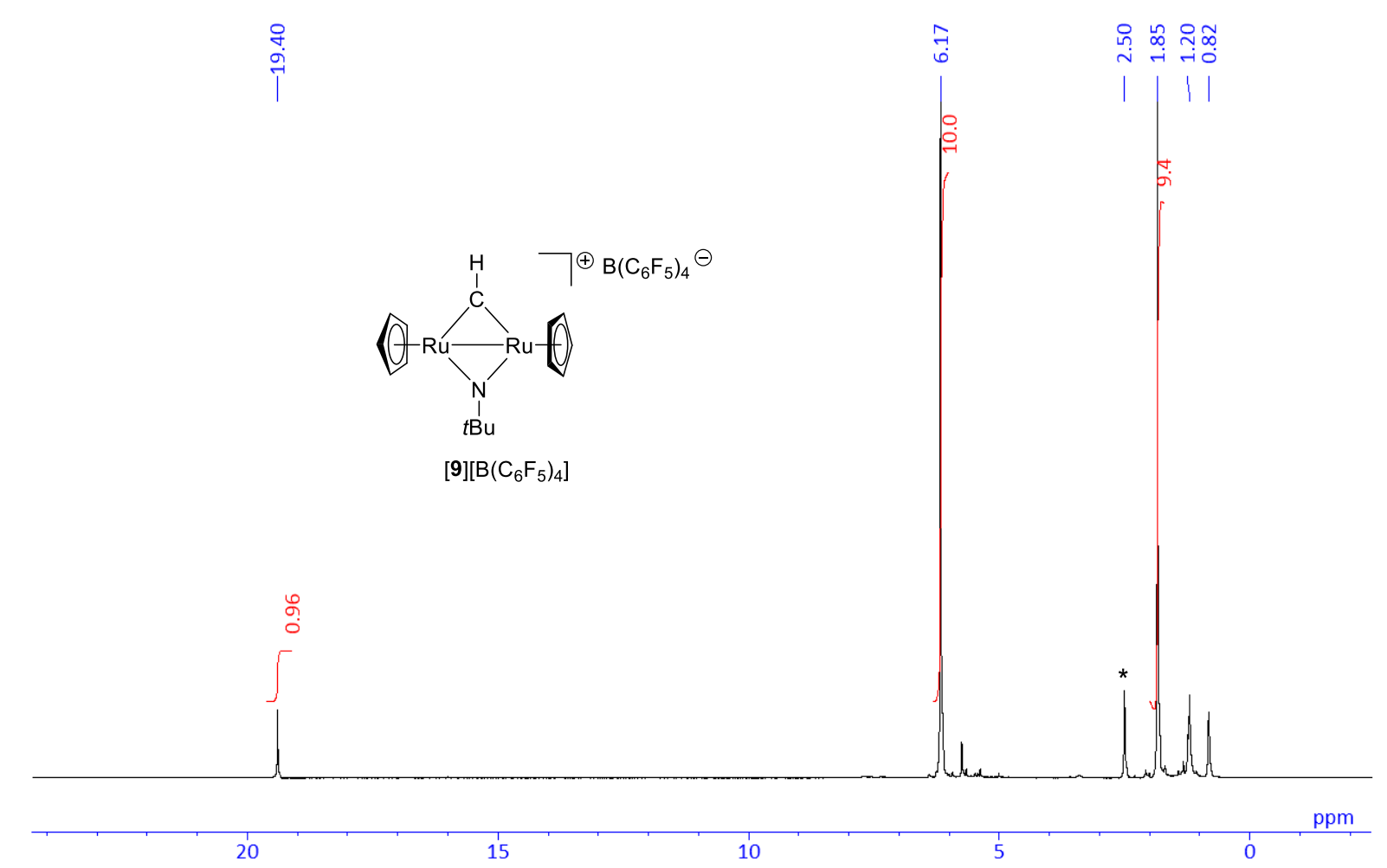

Figure S15. ${ }^{1} \mathrm{H}$ NMR spectrum of $[9]\left[\mathrm{B}\left(\mathrm{C}_{6} \mathrm{~F}_{5}\right)_{4}\right]\left(400 \mathrm{MHz}, \mathrm{DMSO}-\mathrm{d}_{6}\right) . \quad{ }^{*}$ solvent peak

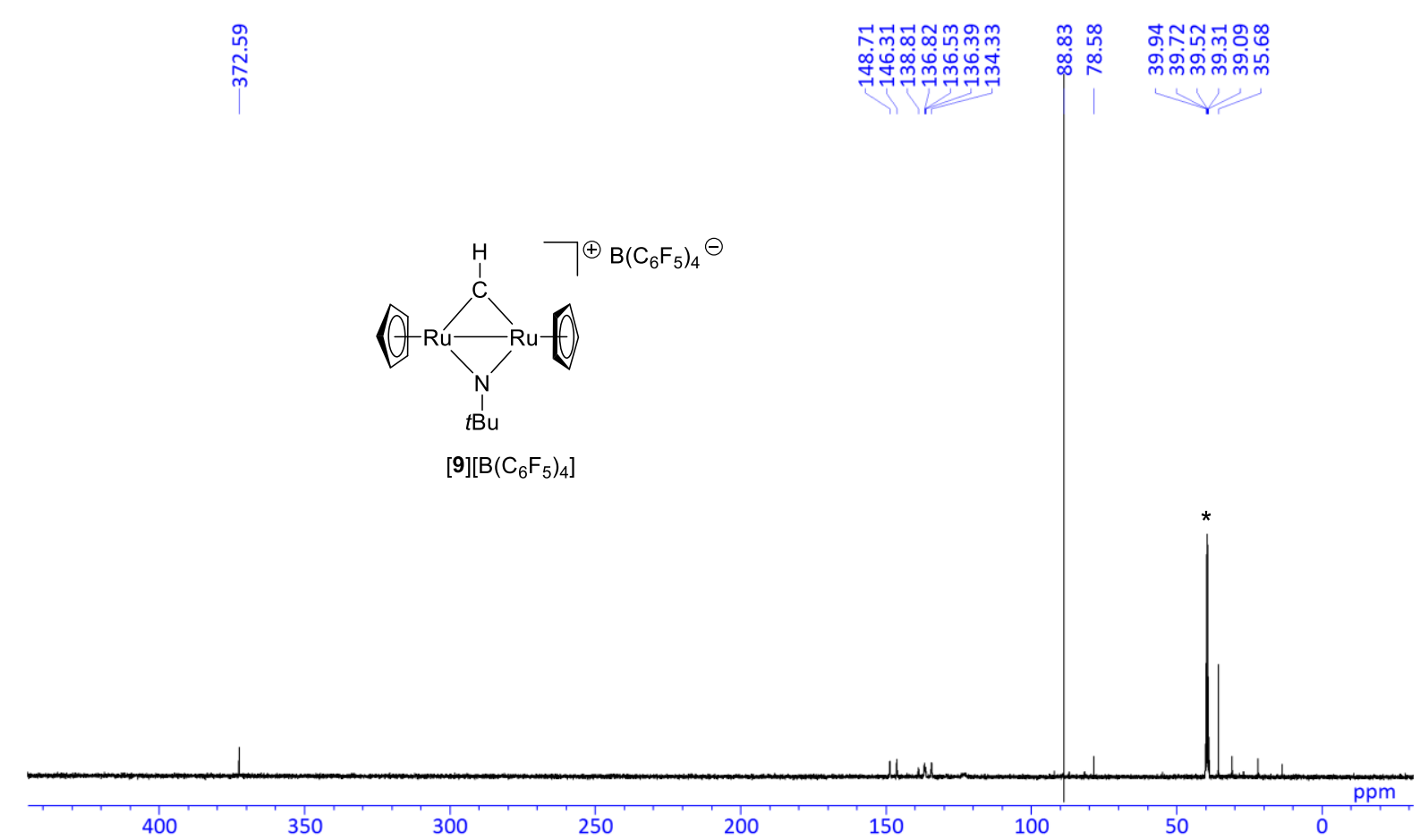

Figure S16. ${ }^{13} \mathrm{C}\left\{{ }^{1} \mathrm{H}\right\}$ NMR spectrum of $[9]\left[\mathrm{B}\left(\mathrm{C}_{6} \mathrm{~F}_{5}\right)_{4}\right]\left(100 \mathrm{MHz}, \mathrm{DMSO}-\mathrm{d}_{6}\right)$. * ${ }^{*}$ solvent peak 


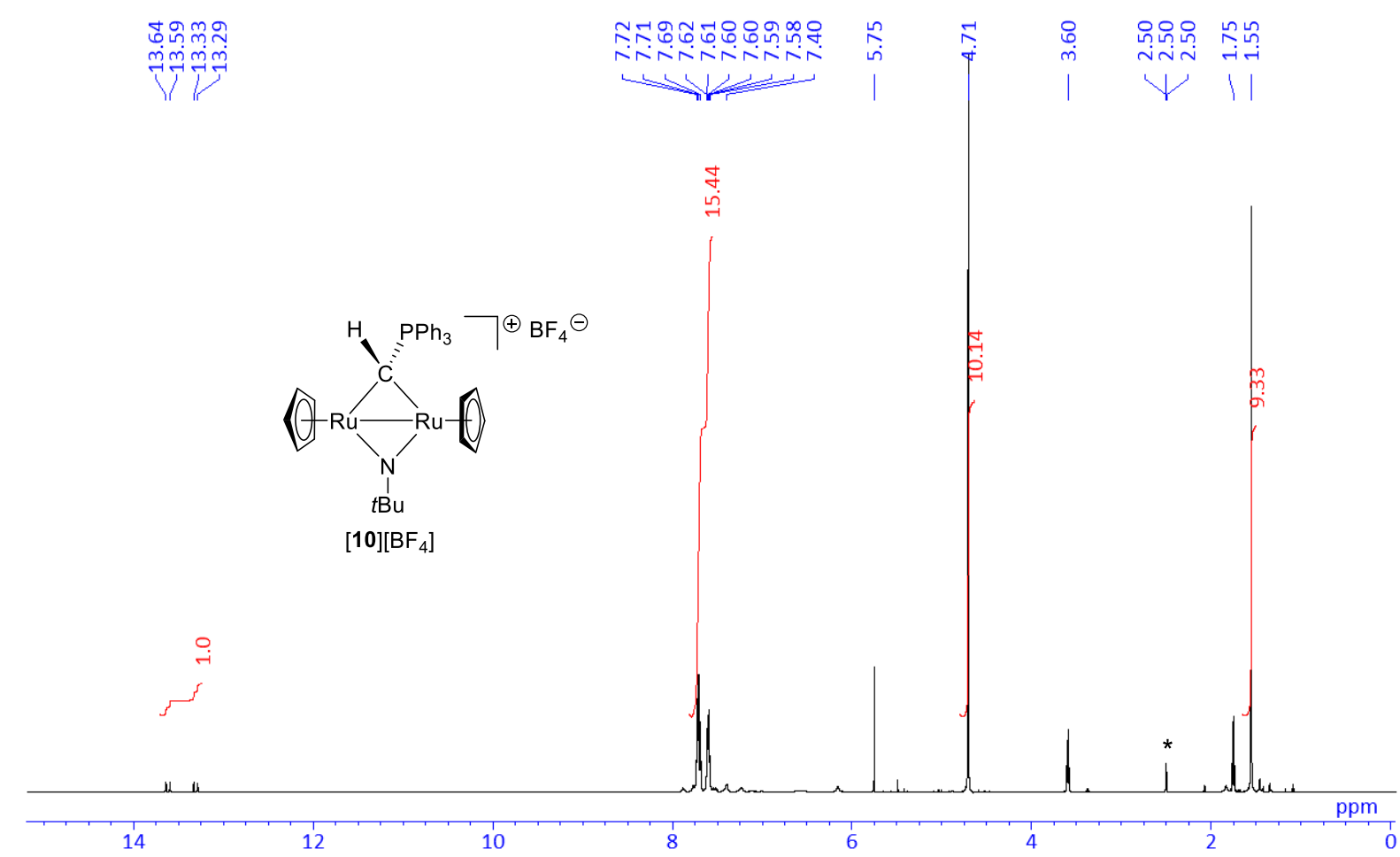

Figure S17. ${ }^{1} \mathrm{H}$ NMR spectrum of $[10]\left[\mathrm{BF}_{4}\right]\left(400 \mathrm{MHz}, \mathrm{DMSO}-\mathrm{d}_{6}\right)$. The sample was ${ }^{13} \mathrm{C}$ enriched at the methylidyne carbon (i.e., $\left.\left[(\mathrm{CpRu})_{2}(\mu-\mathrm{N} t \mathrm{Bu})\left(\mu-{ }^{13} \mathrm{CH}\left(\mathrm{PPh}_{3}\right)\right)\right]\left[\mathrm{BF}_{4}\right]\right) . \quad$ *solvent peak

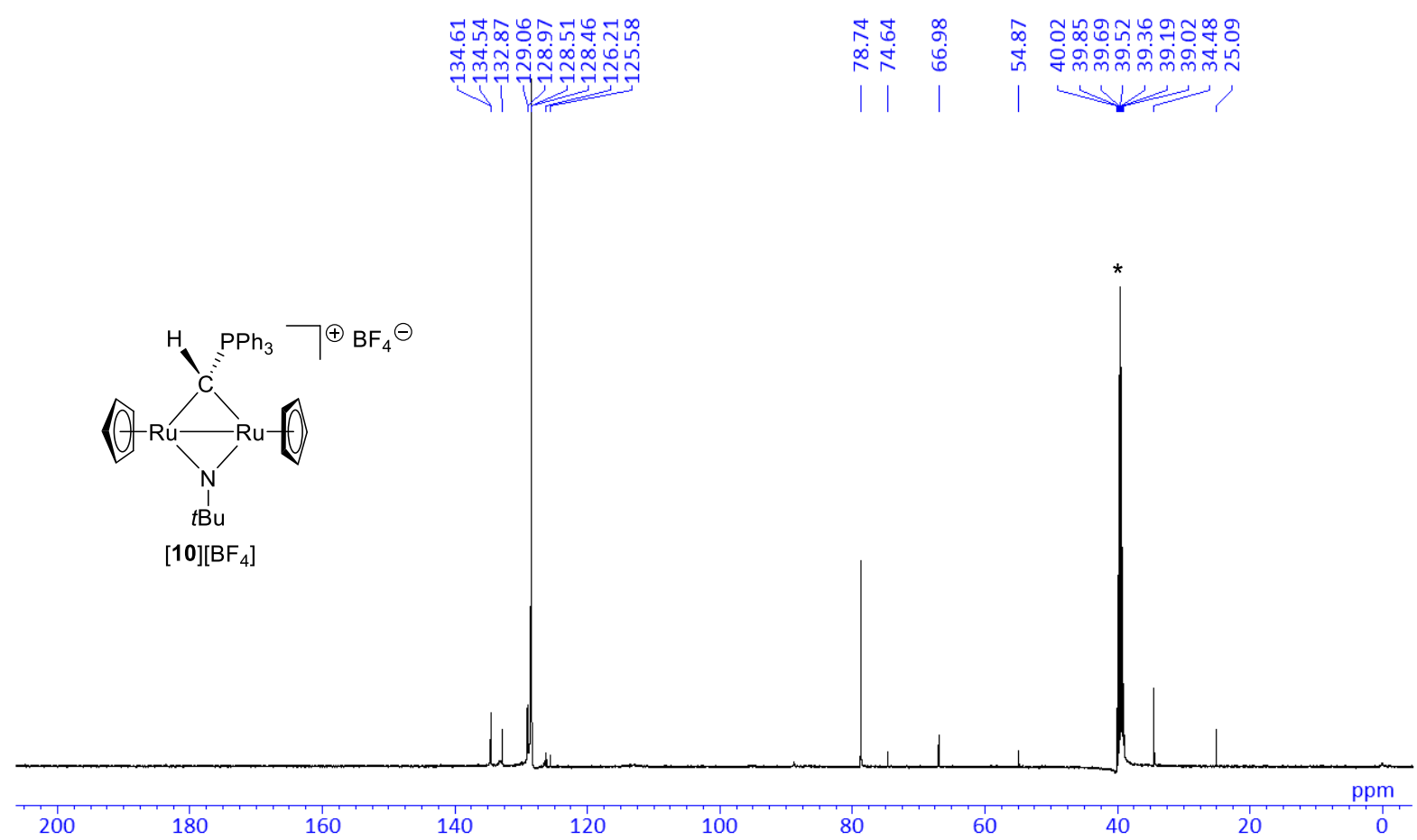

Figure S18. ${ }^{13} \mathrm{C}\left\{{ }^{1} \mathrm{H}\right\}$ NMR spectrum of $[10]\left[\mathrm{BF}_{4}\right]\left(100 \mathrm{MHz}, \mathrm{DMSO}-\mathrm{d}_{6}\right)$. The sample was ${ }^{13} \mathrm{C}$-enriched at the methylidyne carbon (i.e., $\left.\left[(\mathrm{CpRu})_{2}(\mu-\mathrm{N} t \mathrm{Bu})\left(\mu-{ }^{13} \mathrm{CHPPh}_{3}\right)\right]\left[\mathrm{BF}_{4}\right]\right)$. *solvent peak 


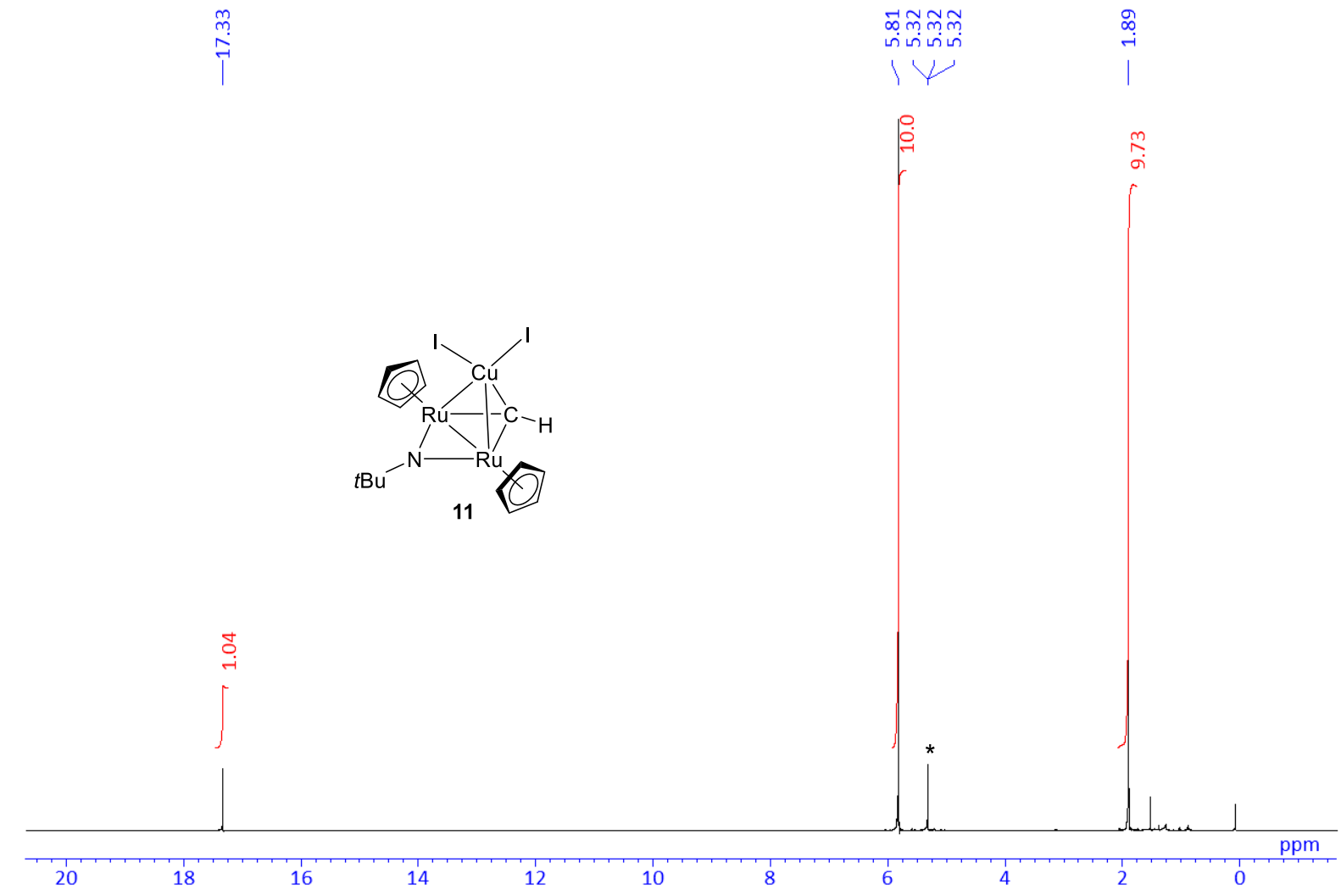

Figure S19. $\quad{ }^{1} \mathrm{H}$ NMR spectrum of $11\left(400 \mathrm{MHz}, \mathrm{CD}_{2} \mathrm{Cl}_{2}\right)$. * * solvent peak

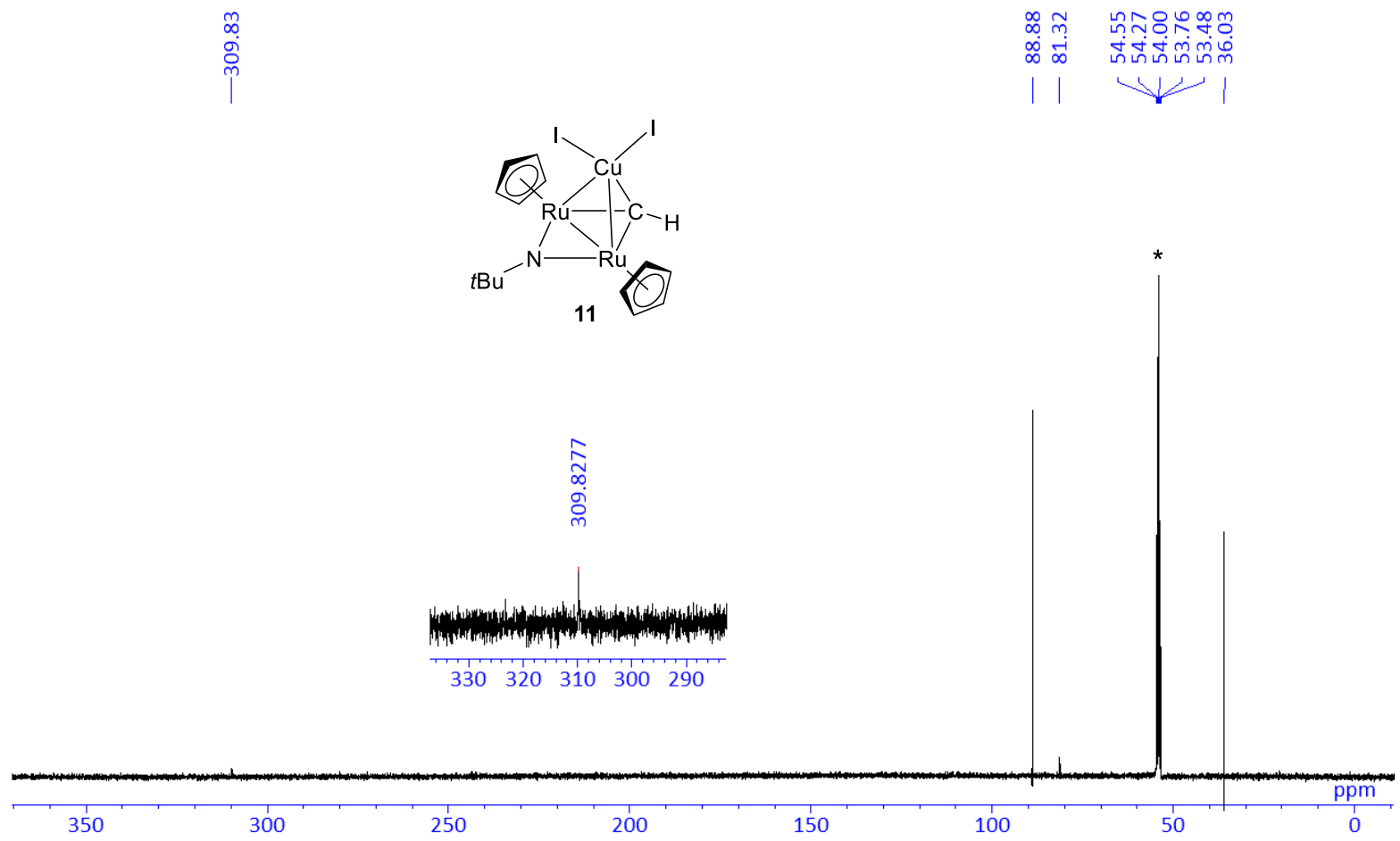

Figure S20. ${ }^{13} \mathrm{C}\left\{{ }^{1} \mathrm{H}\right\}$ NMR spectrum of $11\left(100 \mathrm{MHz}, \mathrm{CD}_{2} \mathrm{Cl}_{2}\right)$. *solvent peak 


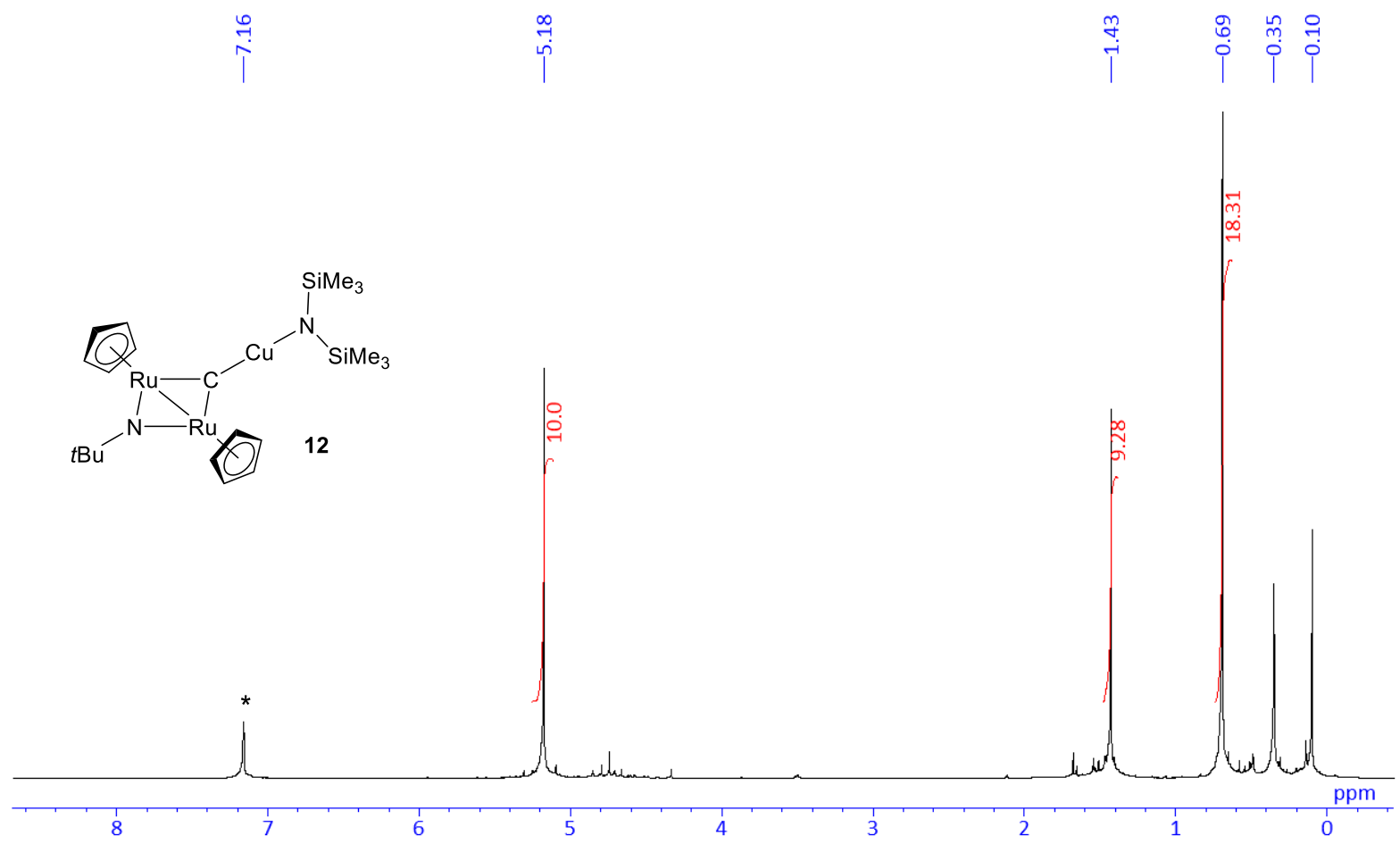

Figure S21. $\quad{ }^{1} \mathrm{H}$ NMR spectrum of $12\left(400 \mathrm{MHz}, \mathrm{C}_{6} \mathrm{D}_{6}\right)$. *solvent peak

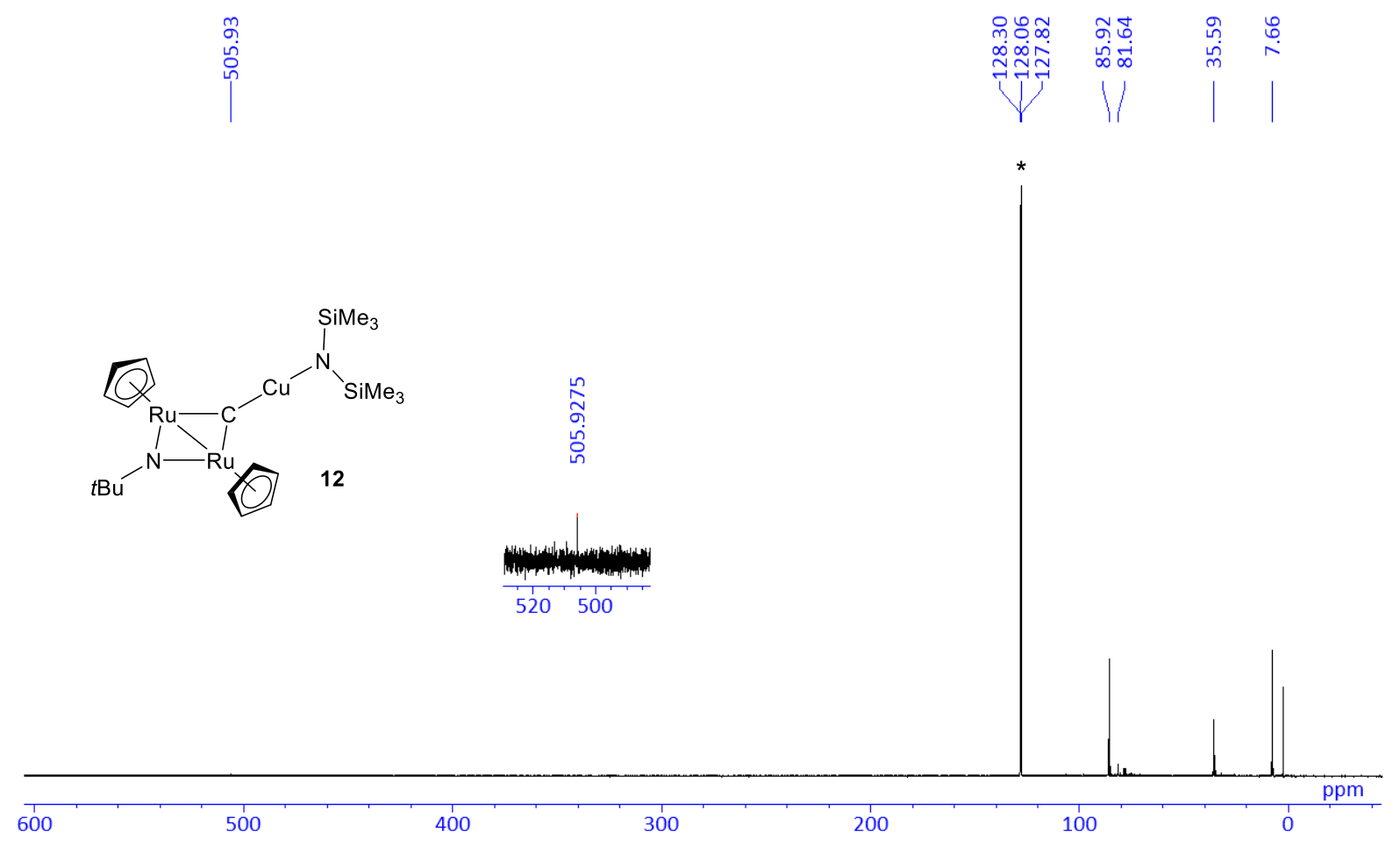

Figure S22. ${ }^{13} \mathrm{C}\left\{{ }^{1} \mathrm{H}\right\}$ NMR spectrum of $12\left(100 \mathrm{MHz}, \mathrm{C}_{6} \mathrm{D}_{6}\right) . \quad{ }^{*}$ solvent peak 


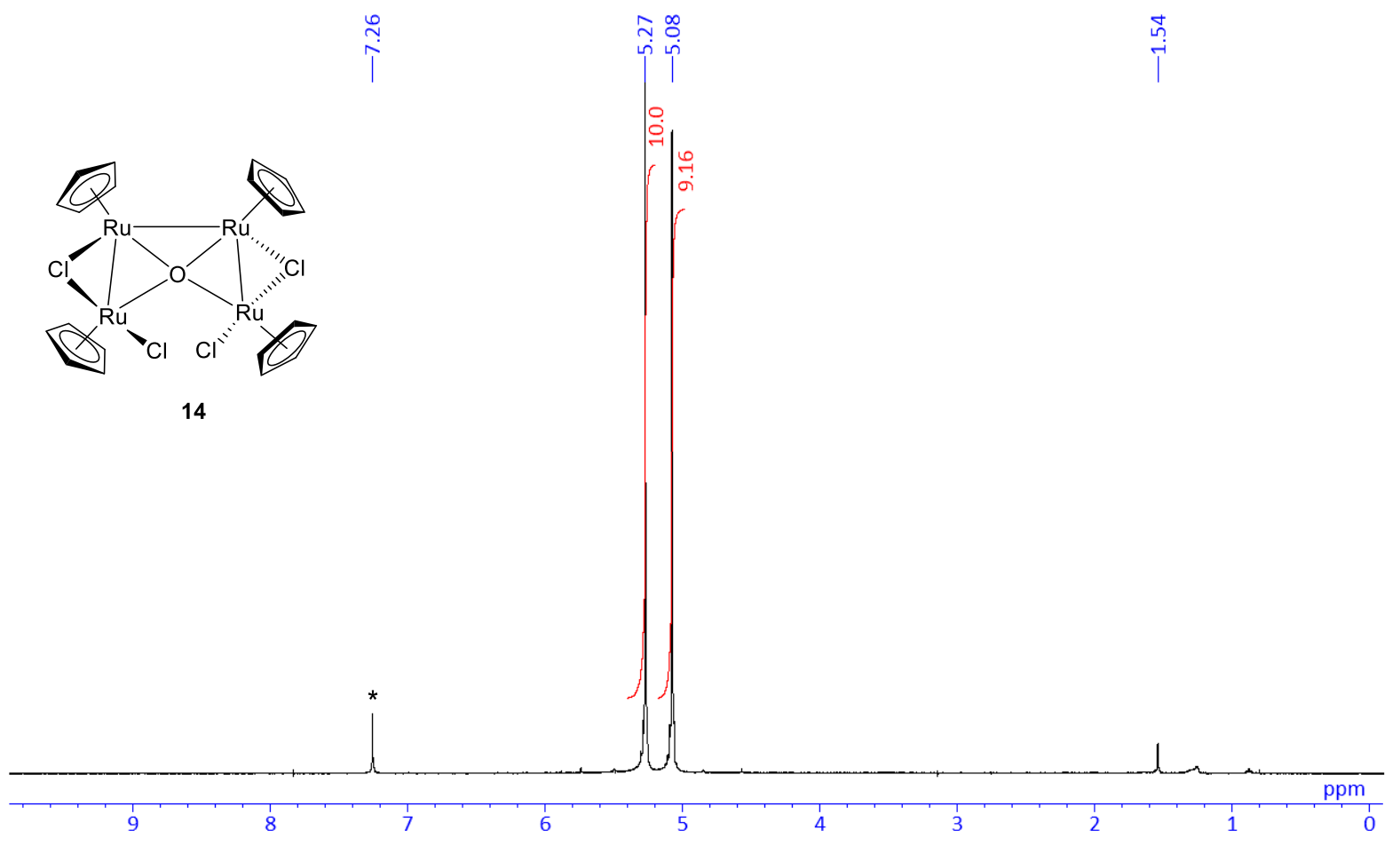

Figure S23. ${ }^{1} \mathrm{H}$ NMR spectrum of $14\left(400 \mathrm{MHz}, \mathrm{CDCl}_{3}\right)$. * ${ }^{\text {solvent peak }}$

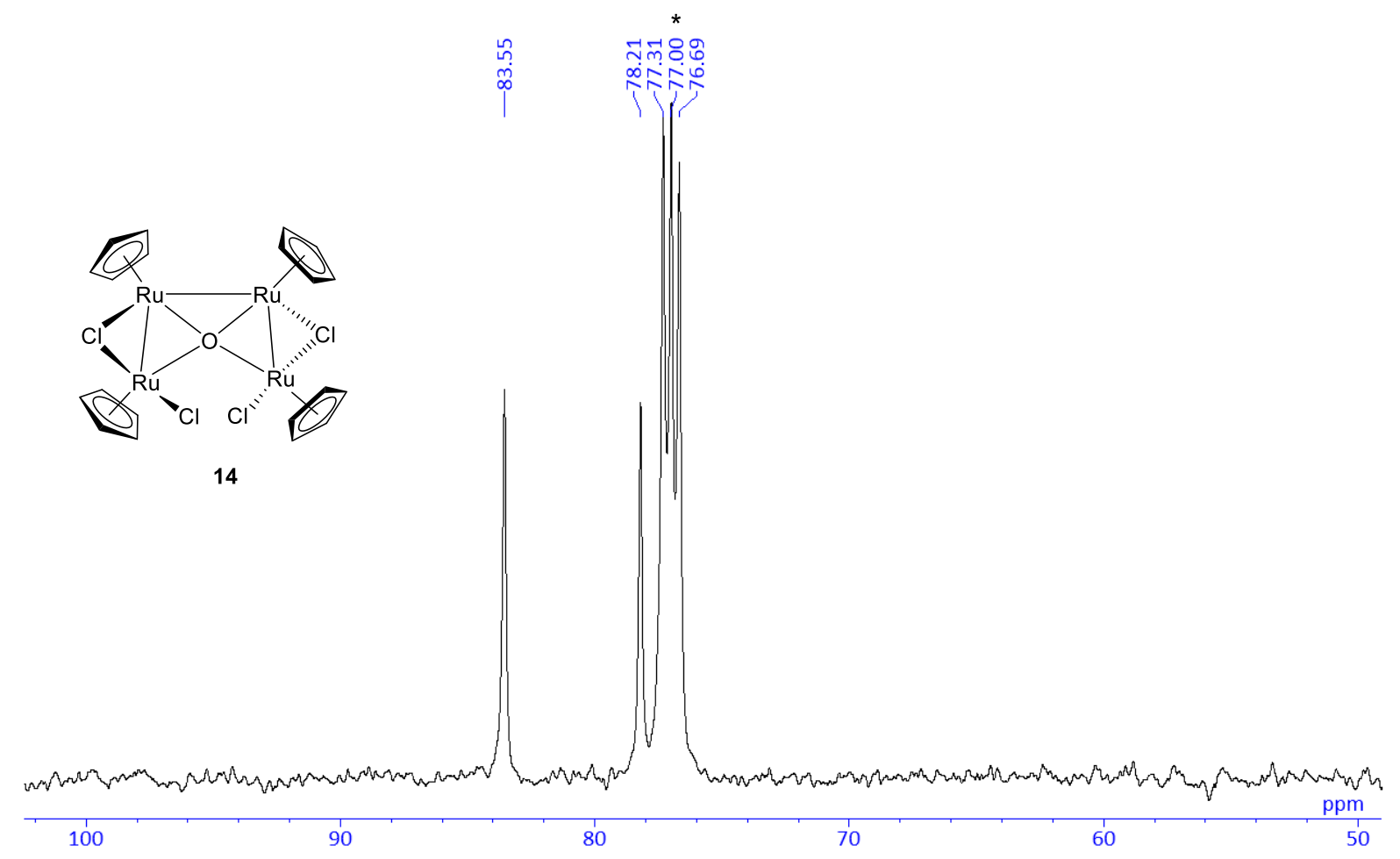

Figure S24. ${ }^{13} \mathrm{C}\left\{{ }^{1} \mathrm{H}\right\}$ NMR spectrum of $14\left(100 \mathrm{MHz}, \mathrm{CDCl}_{3}\right) . \quad$ *solvent peak 


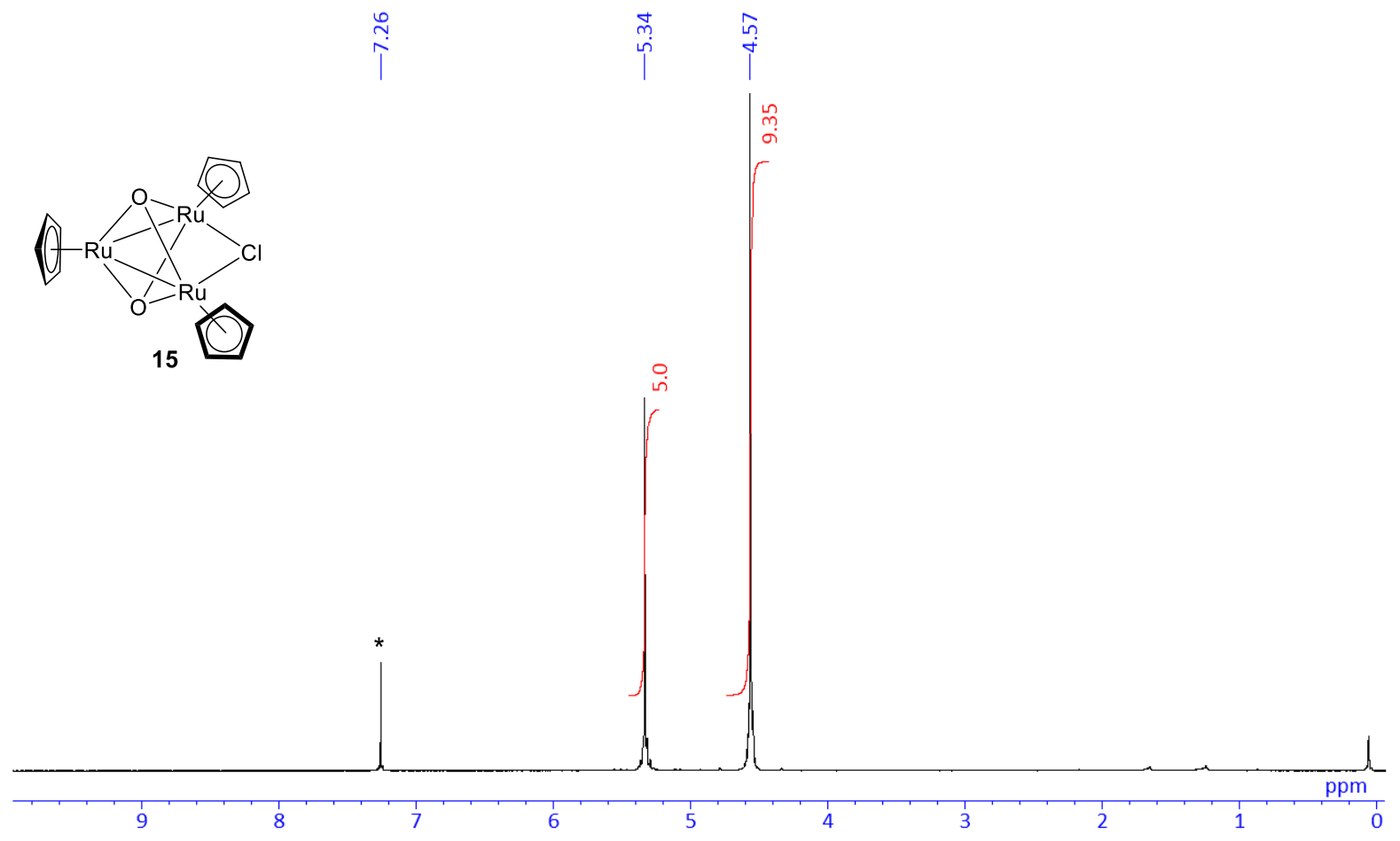

Figure S25. $\quad{ }^{1} \mathrm{H}$ NMR spectrum of $15\left(400 \mathrm{MHz}, \mathrm{CDCl}_{3}\right)$. * solvent peak

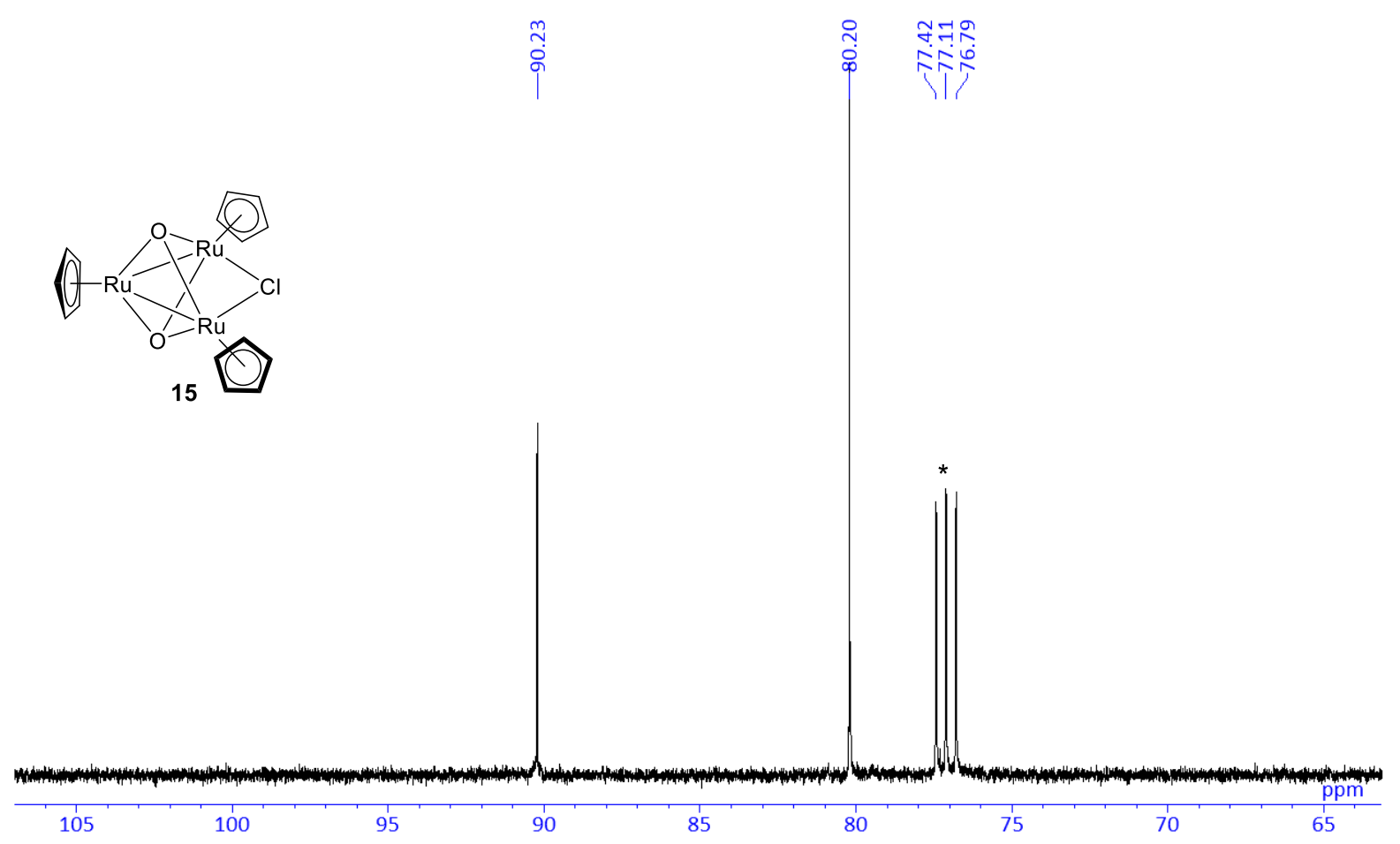

Figure S26. ${ }^{13} \mathrm{C}\left\{{ }^{1} \mathrm{H}\right\}$ NMR spectrum of $15\left(100 \mathrm{MHz}, \mathrm{CDCl}_{3}\right)$. * solvent peak 

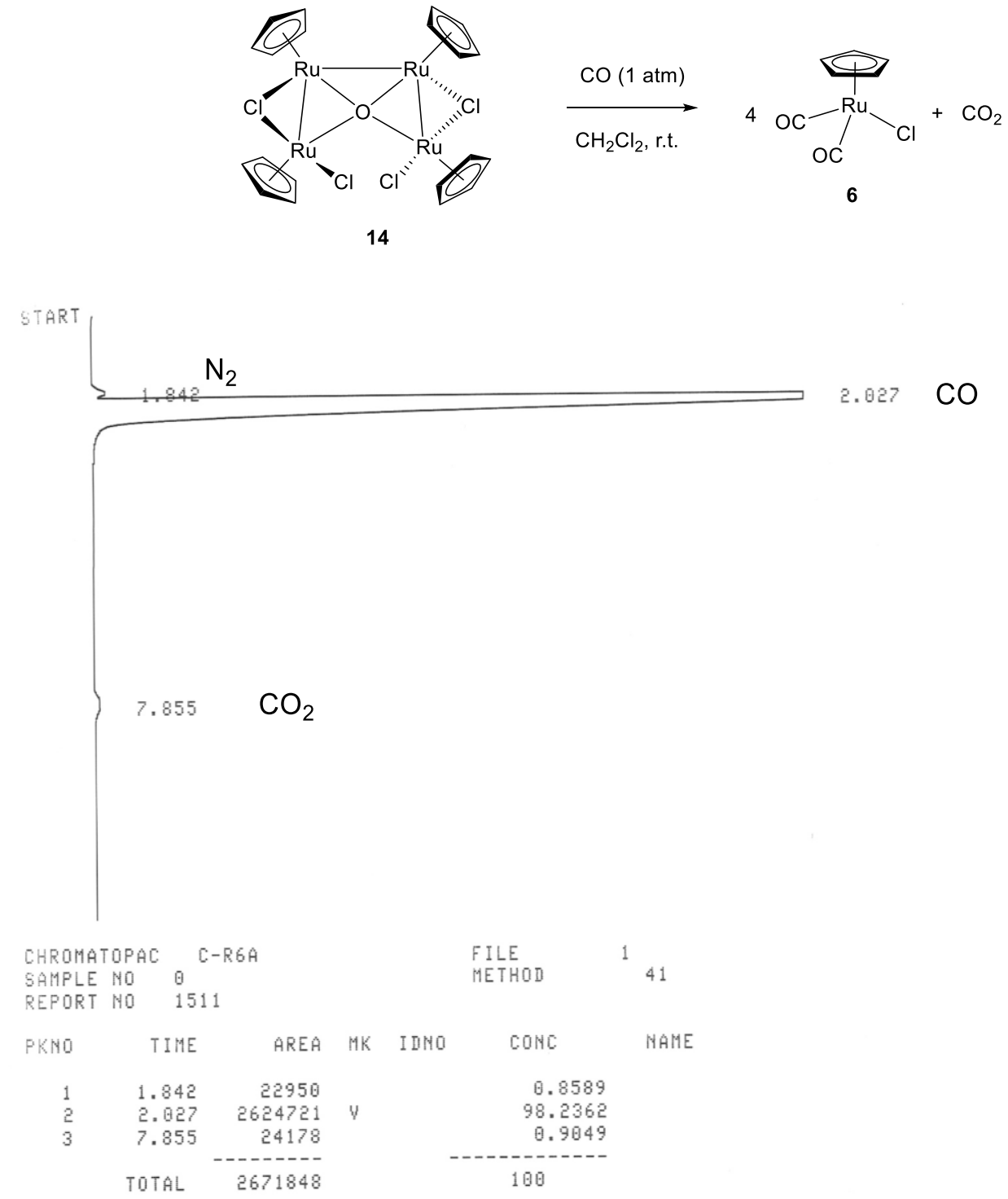

Figure S27. GC for the gas phase analysis of the reaction of $\mathbf{1 4}$ with CO. 
Table S1. Crystallographic data for $[1][2] \cdot \mathrm{CH}_{2} \mathrm{Cl}_{2}, 3$, [9][B( $\left.\left.\mathrm{C}_{6} \mathrm{~F}_{5}\right)_{4}\right] \cdot \mathrm{C}_{6} \mathrm{H}_{14},[9 *]\left[\mathrm{B}\left(\mathrm{C}_{6} \mathrm{~F}_{5}\right)_{4}\right]$.

\begin{tabular}{|c|c|c|c|c|}
\hline & {$[1][2] \cdot \mathrm{CH}_{2} \mathrm{Cl}_{2}$} & 3 & {$[9]\left[\mathrm{B}\left(\mathrm{C}_{6} \mathrm{~F}_{5}\right)_{4}\right] \cdot \mathrm{C}_{6} \mathrm{H}_{14}$} & {$[9 *]\left[\mathrm{B}\left(\mathrm{C}_{6} \mathrm{~F}_{5}\right)_{4}\right]$} \\
\hline formula & $\mathrm{C}_{26} \mathrm{H}_{25} \mathrm{Cl}_{5} \mathrm{Ru}_{3}$ & $\mathrm{C}_{9} \mathrm{H}_{17} \mathrm{ClO}_{2} \mathrm{RuS}_{2}$ & $\mathrm{C}_{42} \mathrm{H}_{27} \mathrm{BF}_{20} \mathrm{NRu}_{2}$ & $\mathrm{C}_{49} \mathrm{H}_{40} \mathrm{BF}_{20} \mathrm{NRu}_{2}$ \\
\hline$M$ & 817.96 & 357.88 & 1138.60 & 1235.77 \\
\hline$T / \mathrm{K}$ & $173(2)$ & $173(2)$ & $173(2)$ & $173(2)$ \\
\hline size $(\mathrm{mm})$ & $0.40 \times 0.10 \times 0.01$ & $0.40 \times 0.30 \times 0.20$ & $0.50 \times 0.20 \times 0.02$ & $0.50 \times 0.20 \times 0.02$ \\
\hline crystal system & monoclinic & orthorhombic & monoclinic & monoclinic \\
\hline space group & $P 2_{1} / c$ & $P 2{ }_{1}{ }_{1}{ }_{2}{ }_{1}$ & $P 2_{1} / c$ & $P 2_{1} / c$ \\
\hline$Z$ & 4 & 4 & 4 & 4 \\
\hline$a(\AA)$ & $10.5084(4)$ & $8.3155(3)$ & $14.8721(7)$ & $13.725(2)$ \\
\hline$b(\AA)$ & $24.7675(9)$ & $10.1479(4)$ & $11.7510(5)$ & $13.765(3)$ \\
\hline$c(\AA)$ & $11.2180(5)$ & $15.8039(6)$ & $23.8266(11)$ & $25.458(5)$ \\
\hline$\alpha(\mathrm{deg})$ & 90.0000 & 90.0000 & 90.0000 & 90.0000 \\
\hline$\beta(\mathrm{deg})$ & $116.625(8)$ & 90.0000 & $102.973(7)$ & 99.381(7) \\
\hline$\gamma(\operatorname{deg})$ & 90.0000 & 90.0000 & 90.0000 & 90.0000 \\
\hline$V\left(\AA^{3}\right)$ & 2610.1(3) & 1333.61(8) & $4057.7(3)$ & $4745.2(15)$ \\
\hline$D_{\text {calc }}\left(\mathrm{g} / \mathrm{cm}^{3}\right)$ & 2.081 & 1.782 & 1.864 & 1.730 \\
\hline$\mu\left(\mathrm{mm}^{-1}\right)$ & 2.241 & 1.668 & 0.871 & 0.752 \\
\hline reflns collected & 24046 & 12797 & 37354 & 45867 \\
\hline unique reflns & 5935 & 3027 & 9232 & 10796 \\
\hline GOF on $F^{2}$ & 1.046 & 1.149 & 1.030 & 1.019 \\
\hline $\mathrm{R} 1[I>2 \sigma(I)]^{a}$ & 0.0349 & 0.0172 & 0.0313 & 0.0484 \\
\hline wR2 (all data) ${ }^{b}$ & 0.0957 & 0.0438 & 0.0779 & 0.1102 \\
\hline
\end{tabular}

${ }^{a} \mathrm{R} 1=(\Sigma \| F \mathrm{o}|-| F \mathrm{c}||) / \Sigma|F \mathrm{o}|,{ }^{b} \mathrm{wR} 2=\left[\left\{\Sigma\left(\mathrm{w}\left(F_{\mathrm{o}}{ }^{2}-F \mathrm{c}^{2}\right)^{2}\right)\right\} / \Sigma \mathrm{w}\left(F_{\mathrm{o}}{ }^{2}\right)^{2}\right]^{1 / 2}$ 
Table S2. Crystallographic data for $[\mathbf{1 0}]\left[\mathrm{BF}_{4}\right] \cdot \mathrm{THF}, \mathbf{1 1}, \mathbf{1 4} \cdot \mathrm{CH}_{2} \mathrm{Cl}_{2}$, and $\mathbf{1 5} \cdot \mathrm{CH}_{2} \mathrm{Cl}_{2}$.

\begin{tabular}{|c|c|c|c|c|}
\hline & {$[\mathbf{1 0}]\left[\mathrm{BF}_{4}\right] \cdot \mathrm{THF}$} & 11 & $\mathbf{1 4} \cdot \mathrm{CH}_{2} \mathrm{Cl}_{2}$ & 15. $\mathrm{CH}_{2} \mathrm{Cl}_{2}$ \\
\hline formula & $\mathrm{C}_{37} \mathrm{H}_{43} \mathrm{BF}_{4} \mathrm{NOPRu}_{2}$ & $\mathrm{C}_{16} \mathrm{H}_{15} \mathrm{Cl}_{3} \mathrm{CuI}_{2} \mathrm{NRu}_{2}$ & $\mathrm{C}_{21} \mathrm{H}_{22} \mathrm{Cl}_{6} \mathrm{ORu}_{4}$ & $\mathrm{C}_{16} \mathrm{H}_{12} \mathrm{Cl}_{3} \mathrm{O}_{2} \mathrm{Ru}_{3}$ \\
\hline$M$ & 837.67 & 847.16 & 907.40 & 645.84 \\
\hline$T / \mathrm{K}$ & $173(2)$ & $173(2)$ & $173(2)$ & $173(2)$ \\
\hline size $(\mathrm{mm})$ & $0.40 \times 0.30 \times 0.20$ & $0.40 \times 0.30 \times 0.30$ & $0.40 \times 0.10 \times 0.02$ & $0.40 \times 0.30 \times 0.01$ \\
\hline crystal system & triclinic & orthorhombic & monoclinic & monoclinic \\
\hline space group & $P-1$ & $P c a 2_{1}$ & $P 2_{1} / c$ & $P 2_{1} / c$ \\
\hline$Z$ & 2 & 4 & 4 & 4 \\
\hline$a(\AA)$ & $10.6170(12)$ & 24.2960 & 18.1695(9) & $12.6009(7)$ \\
\hline$b(\AA)$ & $11.5024(11)$ & 9.6947 & $9.6081(6)$ & $9.9017(5)$ \\
\hline$c(\AA)$ & $14.6273(14)$ & 10.1190 & $15.8204(8)$ & $16.2819(8)$ \\
\hline$\alpha(\operatorname{deg})$ & $93.311(7)$ & 90.0000 & 90.0000 & 90.0000 \\
\hline$\beta(\mathrm{deg})$ & $91.240(6)$ & 90.0000 & $113.606(8)$ & $111.265(8)$ \\
\hline$\gamma(\operatorname{deg})$ & $104.017(7)$ & 90.0000 & 90.0000 & 90.0000 \\
\hline$V\left(\AA^{3}\right)$ & $1729.0(3)$ & 2383.4731 & $2530.7(3)$ & 1893.2(2) \\
\hline$D_{\text {calc }}\left(\mathrm{g} / \mathrm{cm}^{3}\right)$ & 1.609 & 2.361 & 2.381 & 2.266 \\
\hline$\mu\left(\mathrm{mm}^{-1}\right)$ & 0.973 & 5.064 & 2.990 & 2.791 \\
\hline reflns collected & 16378 & 21243 & 23371 & 17384 \\
\hline unique reflns & 7718 & 5339 & 5758 & 4297 \\
\hline GOF on $F^{2}$ & 1.129 & 1.039 & 1.155 & 1.047 \\
\hline $\mathrm{R} 1[I>2 \sigma(I)]^{a}$ & 0.0324 & 0.0211 & 0.0223 & 0.0252 \\
\hline wR2 (all data) ${ }^{b}$ & 0.0943 & 0.0498 & 0.0518 & 0.0599 \\
\hline
\end{tabular}

${ }^{a} \mathrm{R} 1=\left(\Sigma \| F_{\mathrm{O}}|-| F \mathrm{c}||\right) / \Sigma|F \mathrm{o}|,{ }^{b} \mathrm{wR} 2=\left[\left\{\Sigma\left(\mathrm{w}\left(F_{\mathrm{o}}{ }^{2}-F_{\mathrm{c}^{2}}\right)^{2}\right)\right\} / \Sigma \mathrm{w}\left(F_{\mathrm{o}}{ }^{2}\right)^{2}\right]^{1 / 2}$ 